\title{
Variability in porcine microRNA genes and its association with mRNA expression and lipid phenotypes
}

\author{
Emilio Mármol-Sánchez ${ }^{1}$, María Gracia Luigi-Sierra ${ }^{1}$, Anna Castelló1,4, Dailu Guan ${ }^{1}$, Raquel Quintanilla²,
} Raul Tonda ${ }^{3}$ and Marcel Amills $s^{1,4^{*}} \mathbb{D}$

\begin{abstract}
Background: Mature microRNAs (miRNAs) play an important role in repressing the expression of a wide range of mRNAs. The presence of polymorphic sites in miRNA genes and their corresponding 3'UTR binding sites can disrupt canonical conserved miRNA-mRNA pairings, and thus modify gene expression patterns. However, to date such polymorphic sites in miRNA genes and their association with gene expression phenotypes and complex traits are poorly characterized in pigs.
\end{abstract}

Results: By analyzing whole-genome sequences from 120 pigs and wild boars from Europe and Asia, we identified 285 single nucleotide polymorphisms (SNPs) that map to miRNA loci, and 109,724 SNPs that are located in predicted 7mer-m8 miRNA binding sites within porcine $3^{\prime} U T R$. In porcine miRNA genes, SNP density is reduced compared with their flanking non-miRNA regions. By sequencing the genomes of five Duroc boars, we identified 12 miRNA SNPS that were subsequently genotyped in their offspring ( $N=345$, Lipgen population). Association analyses of miRNA SNPs with 38 lipid-related traits and hepatic and muscle microarray expression phenotypes recorded in the Lipgen population were performed. The most relevant detected association was between the genotype of the rs319154814 $(G / A)$ SNP located in the apical loop of the ssc-miR-326 hairpin precursor and PPP1CC mRNA levels in the liver $(q$-value $=0.058)$. This result was subsequently confirmed by qPCR ( $P$-value $=0.027)$. The rs319154814 $(G / A)$ genotype was also associated with several fatty acid composition traits.

Conclusions: Our findings show a reduced variability of porcine miRNA genes, which is consistent with strong purifying selection, particularly in the seed region that plays a critical role in miRNA binding. Although it is generally assumed that SNPs mapping to the seed region are those with the most pronounced consequences on mRNA expression, we show that a SNP mapping to the apical region of ssc-miR-326 is significantly associated with hepatic mRNA levels of the PPP1CC gene, one of its predicted targets. Although experimental confirmation of such an interaction is reported in humans but not in pigs, this result highlights the need to further investigate the functional effects of miRNA polymorphisms that are located outside the seed region on gene expression in pigs.

*Correspondence: marcel.amills@uab.cat

${ }^{1}$ Centre for Research in Agricultural Genomics (CRAG), CSIC-IRTA-UAB-UB, Universitat Autònoma de Barcelona, 08193 Bellaterra, Spain

Full list of author information is available at the end of the article

\section{Background}

Mature microRNA transcripts (miRNAs) are short $(\sim 22 \mathrm{nt})$ non-coding RNAs that play an essential role in the regulation of gene expression [1]. During the biogenesis of miRNAs, one strand of the miRNA duplex binds to the guide-strand channel of an Argonaute protein forming a miRNA-induced silencing complex (miRISC), 
which has the ability to repress mRNA expression by binding to specific $3^{\prime}$ UTR target sites [2]. In postembryonic cells, this repressor mechanism acts mainly by destabilizing the mRNA through decapping and poly(A)tail shortening $[3,4]$ and less often by hindering translation [2]. The binding of the miRNA to its $3^{\prime}$ UTR target site depends critically on the sequence of the seed region, which encompasses nucleotides (nt) 2-8 from the $5^{\prime}$ end of the mature miRNA and interacts with the target site through Watson-Crick pairing [1]. Polymorphisms located within the seed region can affect the proper recognition of mRNA targets [5, 6]. Nevertheless, imperfect seed matches can be compensated by nucleotides 13 to 16 of the mature miRNA, thus providing additional anchor pairing to the seed region [2]. Other sites that are relevant for miRNA processing and function are the basal UG, flanking CNNC and apical UGU motifs [7], as well as a mismatched GHG motif [8], all of which contribute to facilitate miRNA processing [2]. Variability in the $3^{\prime}$ UTR miRNA binding sites can also affect gene expression at the post-transcriptional level $[5,9,10]$.

Evolutionary conservation of miRNAs across species is associated with their level of expression [11, 12] and with the functional importance of the regulatory networks that they modulate [13, 14]. In addition, conservation of the repertoire of mRNAs that are targeted by a given miRNA depends mostly on the age of the associated miRNA gene, with novel miRNAs acquiring targets more rapidly than ancient miRNAs [15]. Conserved sites are particularly enriched in the $5^{\prime}$ end of $3^{\prime}$ UTR [12], although selective pressure on miRNA binding sites is not uniform across miRNA genes [16].

Saunders et al. [5] investigated the variability of 474 human miRNA genes and found that the density of single nucleotide polymorphisms (SNPs) is lower in miRNA loci than in their flanking regions. Moreover, Saunders et al. [5] found that $\sim 90 \%$ of the human miRNA genes do not contain polymorphisms, and that most of the SNPs mapping to miRNAs are located outside the seed region, which provides evidence that the variability of this critical functional motif evolves under strong selective constraints [5]. Indeed, polymorphisms in the first 14 nucleotides of mature miRNAs, and particularly those within the seed region, might abrogate the binding of miRNAs to their $3^{\prime}$ UTR targets, leading to an extensive rewiring of miRNA-mediated regulatory networks [17]. Mammalian miRNA knockouts often display abnormal phenotypes, reduced viability, and clinical disorders [2], although functional redundancy among miRNA family members might mitigate, to some extent, the severity of such manifestations [2]. Polymorphisms within miRNA loci that lie outside the seed region can also affect the processing and stability of miRNAs during their maturation and loading into the functional silencing complex [18, 19]. Moreover, Saunders et al. [5] showed that a broad array of predicted miRNA target sites in the $3^{\prime}$ UTR of mRNAs are polymorphic, which suggests that purifying selection in these regions is less intense than in miRNA genes [17]. Overall, these findings provide evidence for the existence of functional conserved mechanisms of miRNA-mediated gene regulation that are influenced by polymorphic sites at both miRNA loci and their $3^{\prime} U T R$ binding sites [20].

The wild ancestors of pigs were independently domesticated in the Near East and China 10,000 years before present [21]. Then, domestic pigs spread worldwide to become one of the most important sources of animal protein for humans and they have diversified into an extensive array of breeds with distinct morphological and productive features [22]. Pig phenotypes might be explained, at least partially, by modifications in the microRNA-mediated regulation of gene expression [23]. Indeed, several studies have reported associations between SNPs in miRNA binding sites and porcine phenotypic variation [24-29], while fewer studies have investigated the association between SNPs in miRNA genes and complex traits in pigs [30-33]. For instance, Chai et al. [33] reported a significant causal relationship between a SNP in the seed of ssc-miR-378-3p and extensive modifications of its target mRNA repertoire. Moreover, they associated the presence of the observed miRNA seed variant to a structural reconfiguration of the hairpin, leading to an enhanced expression of the mature miRNA [33].

The polymorphism of miRNA genes has not been systematically characterized in pigs despite its potential impact on gene regulation and phenotypic variation. In the current work, our aim was to elucidate the patterns of the variability of miRNA genes in European and Asian wild boars and domestic pigs and to infer whether such patterns are influenced by purifying selection. We also investigated whether SNPs in miRNA genes are associated with liver and muscle gene expression traits and lipid phenotypes recorded in a Duroc pig population.

\section{Methods \\ Characterization of polymorphisms in miRNA genes and their $3^{\prime} U T R$ binding sites in a worldwide sample of pigs and wild boars \\ Retrieval of porcine whole-genome sequences}

Whole-genome sequences (WGS) from 120 wild boars and domestic pigs (Sus scrofa) were retrieved from the NCBI Sequence Read Archive (SRA) database (https:// www.ncbi.nlm.nih.gov/sra). Most of these WGS have been reported in previous publications [34-37] and detailed information is available in Additional file 1: 
Table S1. The 120 WGS corresponded to Asian domestic pigs ( $A D M, N=40)$, Asian wild boars (AWB, $N=20$ ), European domestic pigs (EDM, N=40) and European wild boars (EWB, $N=20)$. The ADM group included WGS from Meishan, Tongchen, Jinhua, Rongchan, Wuzhishan, Tibetan, Sichuan, Hetao, Minzhu, Bamaixang and Laiwu pigs, and the EDM group was composed of WGS from Pietrain, Mangalitza, Iberian, Duroc, American Yucatan (from America, but with a European origin), Yorkshire, Landrace, Hampshire and Large-white pigs. Both AWB and EWB samples were selected according to their geographic distribution (see Additional file 1: Table S1). The EWB group included one sample from the Near East. Raw data in SRA format were downloaded from SRA public repositories and converted into fastq format by using the fastq-dump 2.8.2 tool that is available in the SRA-toolkit package (https://ncbi.github.io/ sra-tools/).

\section{Whole-genome sequence data processing and calling of single nucleotide polymorphisms}

Fastq paired-end files generated from SRA data were filtered according to their quality, and sequence adapters were trimmed by using the Trimmomatic software (v.0.36) with default parameters [38]. Trimmed pairedend sequences were aligned against the Sus scrofa reference genome (Sscrofa11.1) [39] with the BWA-MEM algorithm [40] and default settings. Sequence alignment map (SAM) formatted files were sorted and transformed into binary (BAM) formatted files. PCR duplicates were subsequently identified with the MarkDuplicates package from the Picard tool (https://broadinstitute.github.io/pic$\operatorname{ard} /$ ) and removed to perform insertion-deletion (InDel) realignment with the IndelRealigner package from the Genome Analysis Toolkit (GATK v.3.8) [41]. Base quality score recalibration (BQSR) was implemented with the BaseRecalibrator package from GATK v.3.8. Variant calling was implemented with the HaplotypeCaller function according to GATK best practices [41]. Individual gVCF formatted files, including both polymorphic and homozygous blocks, were generated with the GenotypeGVCFs package from the GATK v.3.8 tool [41], and they were merged into separate multi-individual variant calling format (VCF) files containing single polymorphic and InDel sites for each defined population (i.e. ADM, EDM, AWB and EWB). Finally, variants were distributed into SNP and InDel files with the SelectVariants package and quality-filtered using the FilterVariants function from GATK v.3.8 [41]. For SNPs, we used the following parameters: $\mathrm{QD}<2.0, \mathrm{FS}>60.0, \mathrm{MQ}<40.0, \quad \mathrm{MQRankSum}<-12.5$, ReadPosRankSum $<-8.0$, SOR $>3.0$. In the current study, we did not take InDels into consideration because the reliability and reproducibility of the detection of InDels based on commonly used pipelines for variant calling are low across tools and analyzed datasets [42, 43].

\section{Identification of single nucleotide polymorphisms in miRNA genes and in their $3^{\prime}$ UTR binding sites}

Single nucleotide polymorphisms mapping to annotated porcine miRNA loci $(\mathrm{N}=370)$ were retrieved by using the curated Sscrofa11.1 annotation for miRNA regions available in the miRCarta v1.1 [44] and miRBase [45] databases. In addition, annotated mature miRNA loci $(\mathrm{N}=409)$ within miRNA genes $(\mathrm{N}=370)$ were retrieved, and SNPs located in the mature and seed regions (positions 2 to 8 at the $5^{\prime}$ end of the mature miRNA) were identified. A comprehensive description of miRNA gene regions and miRNA-mRNA interactions is shown in Fig. 1a, b.

We also retrieved the annotated 3'UTRs from porcine mRNA transcripts available at Ensembl repositories (v.100, http://www.ensembl.org/info/data/ftp/ index.html) and the corresponding set of sequences was interrogated against the seed regions of the 409 porcine mature miRNAs considered in our study. Seed sequences were reverse-complemented and searched along the $3^{\prime} \mathrm{UTR}$ sequences of mRNA genes by using the locate function of the SeqKit toolkit [46]. All 7mer$\mathrm{m} 8$ canonical seed pairing sites and the 8 mer seed pairing sites (Fig. 1) were identified for each miRNA seed, and, based on this information, the corresponding putative miRNA-mRNA target pairs were established. Additional non-canonical miRNA-mRNA interactions were not considered (Fig. 1b) as they are regarded as less biologically significant than $7 \mathrm{mer}-\mathrm{m} 8$ or 8 mer interactions $[2,47,48]$. Subsequently, the genomic locations of both 7 mer-m 8 and 8 mer matching regions were determined, and SNPs that were located in such predicted miRNA binding sites were identified.

Principal components analyses (PCA) were performed with the smartPCA software [49]. These PCA analyses were based on: (1) autosomal whole-genome SNPs, (2) autosomal SNPs from miRNA genes, (3) autosomal SNPs from $3^{\prime} \mathrm{UTR}$, or (4) autosomal SNPs from 8mer and $7 \mathrm{mer}-\mathrm{m} 83^{\prime} \mathrm{UTR}$ sites. When implementing the PCA based on whole-genome data, SNPs that complied with the following parameters were retained: (1) minimum allele frequency $>0.05$, and (2) Hardy-Weinberg equilibrium exact test $P$-value $>0.001$. In contrast, no filtering was performed in the PCA based on miRNA SNPs because of their small number.

\section{Frequency and distribution of SNPs in miRNA genes}

To investigate the distribution of SNPs along miRNA genes, SNPs were classified according to their location, 


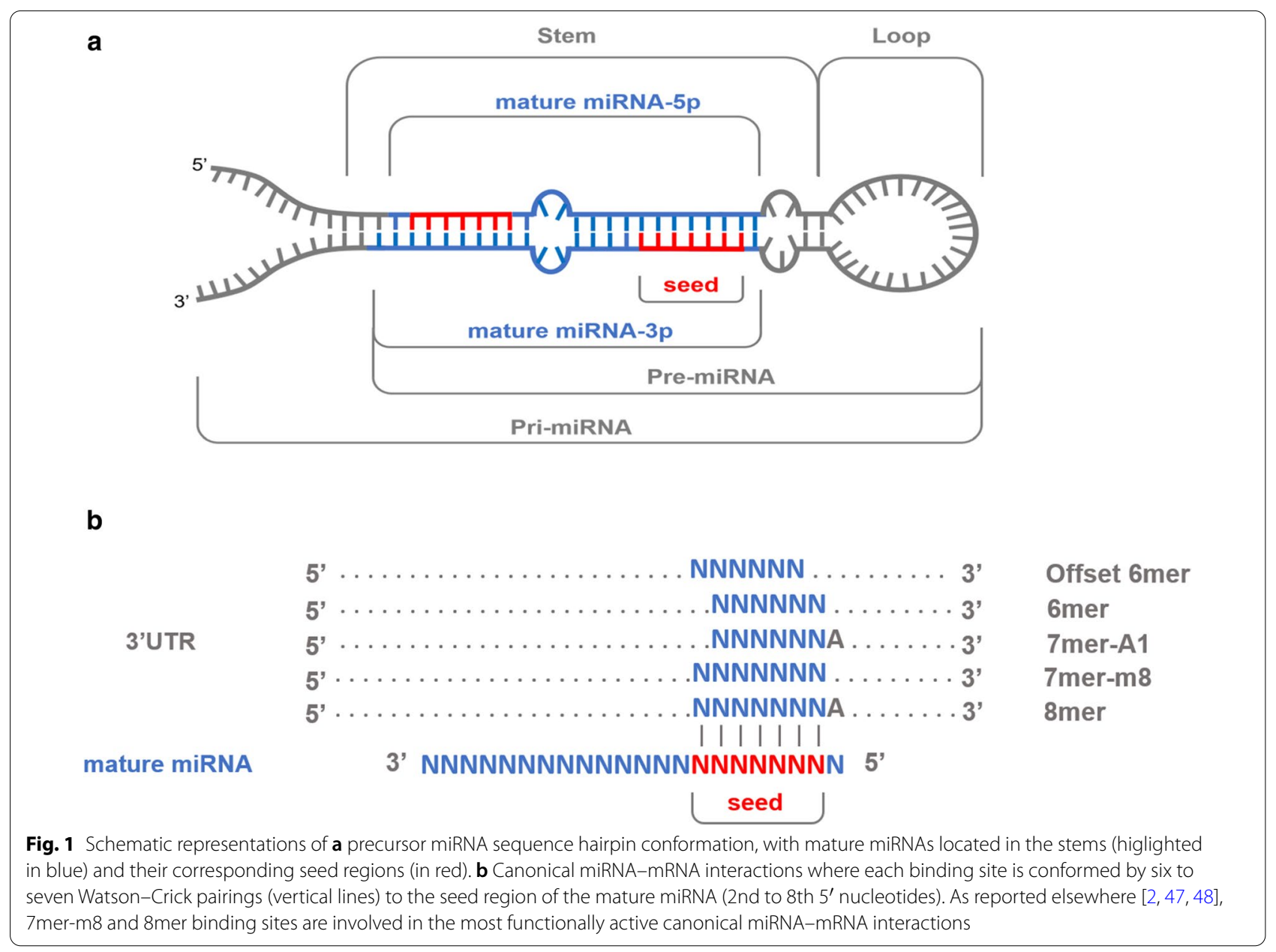

i.e., SNPs within the seed region of mature miRNAs were flagged as "seed", whereas SNPs located in mature miRNAs but outside the seed were classified as "mature". In addition, SNPs classified as "mature" were assigned to the following subtypes: (1) "anchor" (1st position at the $5^{\prime}$ end), and (2) "supplemental pairing" (position 13 to 18 from the $5^{\prime}$ end). The remaining SNPs were assigned to the "precursor" class. Allele frequencies were estimated in the whole set of porcine samples $(\mathrm{N}=120)$ and in each EDM, ADM, EWB and AWB groups independently (see Additional file 2: Table S2).

In order to calculate the SNP density in precursor, mature and seed miRNA regions, first we calculated the total nucleotide length of each of these regions in the set of analyzed porcine miRNA loci. Seed length was calculated by considering seven nucleotide positions in each of the annotated mature miRNAs $(\mathrm{N}=409)$ mapping to the 370 miRNA loci considered in our study (7 bp $\times 409=2863 \mathrm{bp}$ ). The total length of the 409 mature miRNAs was determined $(\sim 22 \mathrm{bp} \times 409=8916 \mathrm{bp})$, and the total seed length (2863 bp) was subtracted from $8916 \mathrm{bp}$ to obtain the mature length (6053 bp), which corresponds to the total length of all mature miRNA sequences excluding the seeds. The precursor length $(22,229 \mathrm{bp})$ was calculated by subtracting the seed length (2863 bp) and mature length $(6053 \mathrm{bp})$ from the total length $(31,145 \mathrm{bp})$ of all miRNA loci $(\mathrm{N}=370)$. Then, the SNP density $(D)$ in each of these regions was calculated as follows:

$$
D=\frac{N_{r} \times 100}{L_{r}}
$$

where $N_{r}$ is the total number of miRNA SNPs $(N=285)$, or the number of SNPs detected in each of the defined regions, (i.e., 221, 52 and 12 SNPs in precursor, mature and seed regions, respectively); $L_{r}$ is the total nucleotide length of all miRNA loci $(31,145 \mathrm{bp})$, or the total number of nucleotides in miRNA loci belonging to the "precursor" (22,229 bp), "mature" (6053 bp) or "seed" (2863 bp) regions. These calculations yield the number of SNPs per bp for each category, but we decided to adjust this estimate to a 100-bp window, which results 
in the multiplication of $N_{r}$ by 100 in the above formula. We also compared the SNP density between miRNA loci $(N=370)$ and their flanking genomic regions $( \pm 1 \mathrm{~kb})$ by considering 10 upstream and downstream nucleotide bins of 100 bp each. Differences in SNP density across miRNA loci, as well as its comparison with surrounding regions, were estimated with a non-parametric approach using a Mann Whitney U test [50].

\section{Investigating the association of miRNA polymorphisms with gene expression and phenotype data recorded in Duroc pigs \\ Whole-genome sequencing of five Duroc pigs}

In 2003, five Duroc boars were selected and used as founders of a half-sib population of purebred Duroc pigs that are dedicated to the production of high-quality cured ham. By whole-genome sequencing of these five individuals, our aim was to identify segregating miRNA SNPs and investigate their association with gene expression traits and lipid phenotypes recorded in the offspring of the five boars (Lipgen population, $\mathrm{N}=350$ ). Genomic DNA was extracted [51] and sequenced at the Centre Nacional d'Anàlisi Genòmica (CNAG, Barcelona, Spain). Paired-end multiplex libraries were prepared according to the instructions of the manufacturer with the KAPA PE Library Preparation kit (Kapa Biosystems, Wilmington, MA). Libraries were loaded to Illumina flow-cells for cluster generation prior to producing 100-bp pairedend reads on a HiSeq2000 instrument following the Illumina protocol. Base calling and quality control analyses were performed with the Illumina RTA sequence analysis pipeline according to the instructions of the manufacturer. Quality-checked filtered reads were mapped to the Sus scrofa genome version 11.1 and processed for SNP calling according to GATK best practice recommendations [41] and following the same protocol described in the previous section entitled "Whole-genome sequence data processing and calling of single nucleotide polymorphisms". Since only five boars were sequenced, SNPs within miRNA loci were retrieved without applying quality filtering criteria, in order to maximize the number of identified miRNA SNPs.

\section{Recording of phenotypes related with gene expression and lipid traits in the Lipgen population}

In total, 350 Duroc barrows, sired by the five Duroc founder boars mentioned above, were used as a resource population (Lipgen population $[52,53]$ ) to evaluate associations between miRNA SNPs and phenotypes related with gene expression and lipid traits. The five sequenced boars were mated with 400 sows on three farms and one offspring per litter was selected for phenotypic recording (only 350 individuals provided valid records). All selected piglets were weaned, castrated and subsequently fattened in four contemporary batches on the IRTA pig experimental farm in Monells (Girona, Spain) under intensive standard conditions. Once they reached $\sim 122 \mathrm{~kg}$ of live weight ( 190 days of age), they were slaughtered in a commercial abattoir following the guidelines of the current Spanish legislation (https://www.boe.es/buscar/ doc.php?id=BOE-A-1995-3942). After slaughtering, tissue samples from the gluteus medius (GM) and longissimus dorsi (LD) skeletal muscles and liver were obtained according to previously described methods [54-56].

Total DNA was extracted from each sample following Vidal et al. [51]. In total, 345 DNA samples from the initial set of 350 pigs were successfully obtained and processed for further genotyping. Total RNA was extracted from $\mathrm{GM}(\mathrm{N}=89$ pigs $)$ and liver $(\mathrm{N}=87$ pigs $)$ tissue samples by using the Ribopure RNA isolation kit (Ambion, Austin, TX). Expression mRNA profiles were characterized by hybridizing total RNA to GeneChip porcine arrays (Affymetrix Inc., Santa Clara, CA), which encompass 23,998 probes $[54,57]$. Further details about tissue collection, sample selection, RNA isolation and microarray hybridization procedures are in Cánovas et al. [54]. Microarray data pre-processing, background correction, normalization and $\log _{2}$-transformation of expression estimates were performed with a robust multi-array average method per probe [58]. The mas5calls function from the affy $R$ package [58] was then applied to identify probes with intensity signals that were higher than the background noise. This function applies a Wilcoxon signed rank-based gene expression presence/absence detection algorithm for labeling expressed probes in each sample. Control probes and those with expression levels below the detection threshold in more than 50\% of samples were discarded from further analyses. As a result, we retained only the probes which corresponded to mRNAs that were robustly expressed in GM muscle $(12,131$ probes) and liver (12,787 probes) tissues. Probes from the GeneChip Porcine Genomic array identifiers (Affymetrix, Inc., Santa Clara, CA) of GM skeletal muscle and liver tissues were assigned to mRNA genes annotated in the Sscrofa11.1 assembly [39] using the BioMart tool [59].

With regard to lipid-related phenotypes, 38 traits were measured in the Duroc Lipgen population, i.e. backfat thickness measured between the 3rd and 4th ribs, backfat thickness at the last rib, ham fat thickness, and intramuscular fat content and composition of GM and LD skeletal muscle samples $(\mathrm{N}=345)[53,55]$ (see Additional file 3: Table S3). Briefly, intramuscular fatty acid (IMF) content in the GM and LD muscles was measured with the Near Infrared Transmittance technique (NIT, Infratec 1625, Tecator Hoganas, Sweden), whereas muscle cholesterol 
measurements were inferred following Cayuela et al. [60]. Gas chromatography of methyl esters was used to determine muscle fatty acids (FA) composition, including the percentages of saturated (SFA), unsaturated (UFA), monounsaturated (MUFA) and polyunsaturated (PUFA) fatty acids. Live and carcass weights (used as covariates in the statistical analyses), as well as backfat and ham fat thickness, were measured on a regular basis, prior (live weight and backfat thickness) and after slaughter (carcass weight and ham fat thickness). Mean and standard deviations of lipid traits recorded in the Lipgen population $(\mathrm{N}=345)$ are described in Additional file 3: Table S3.

\section{Genotyping of a panel of single nucleotide polymorphisms mapping to microRNA genes in the Lipgen population}

Twelve SNPs that mapped to miRNA loci and segregated in the five sequenced parental Duroc boars were genotyped in the 345 Duroc pigs from the Lipgen population (Table 1). Briefly, selected miRNA SNPs and their flanking regions (60 bp upstream and downstream) were used for the assay design with the Custom TaqMan Assay Design Tool website (https://www5.appliedbiosystems. com/tools/cadt/; Life Technologies). Genotyping was carried out at the Servei Veterinari de Genètica Molecular of the Universitat Autònoma of Barcelona (http://sct. uab.cat/svgm/en) by using a QuantStudio 12K Flex RealTime PCR System (Thermo Fisher Scientific, Barcelona, Spain).

\section{Association analyses between miRNA SNPs and mRNA expression and lipid phenotypes}

Genotype data corresponding to the 12 miRNA SNPs mentioned above (Table 1) were processed with the
PLINK software [61] in order to generate formatted files for subsequent analyses. The Genome-Wide Efficient Mixed-Model Association (GEMMA) software [62] was used to implement association analyses between genotyped SNPs and phenotypes related with microarray gene expression data and lipid traits. The following univariate mixed model was used:

$$
\mathbf{y}=\mathbf{W} \alpha+\mathbf{x} \delta+\mathbf{u}+\boldsymbol{\varepsilon},
$$

where $\mathbf{y}$ is the vector of recorded phenotypes for each individual; $\alpha$ is a vector indicating the intercept plus the considered fixed effects, i.e., batch effect with four categories (all traits), farm of origin effect with three categories (all traits) and laboratory of processing with two categories (GM and liver microarray expression data). $\alpha$ also includes the following covariates: IMF of LD (for LD fatty acid composition traits), IMF of GM (for GM fatty acid composition traits), live weight (for backfat thickness) and carcass weight (for ham fat thickness); $\mathbf{W}$ is the incidence matrix relating phenotypes with their corresponding effects; $\mathbf{x}$ is the vector of genotypes for the 12 miRNA polymorphisms; $\delta$ is the allele substitution effect for each polymorphism; $\mathbf{u}$ is a vector of random individual genetic effects with an $n$-dimensional multivariate normal distribution $\mathrm{MVN}_{n} \sim\left(0, \lambda \tau^{-1} \mathbf{K}\right)$, where $\tau^{-1}$ is the variance of the residual errors, $\lambda$ is the ratio between the two variance components and $\mathbf{K}$ is the known relatedness matrix derived from SNP information; and $\varepsilon$ is the vector of residual errors.

Association analyses were performed between each miRNA polymorphism (Table 1) and the 38 lipid-related traits listed in Additional file 3: Table S3. Moreover, we explored the associations between miRNA SNPs and

Table 1 List of miRNA polymorphisms genotyped in a population of Duroc pigs ( $N=345$, Lipgen population)

\begin{tabular}{|c|c|c|c|c|c|c|c|c|}
\hline microRNA & $\mathrm{SSC}^{\mathrm{a}}$ & Start (bp) & End (bp) & Strand & SNP & Type & Alt. allele & Frequency $^{c}$ \\
\hline ssc-miR-339 & 1 & $164,025,972$ & $164,026,057$ & - & rs81349391 & Apical loop & G & 0.4138 \\
\hline ssc-miR-130a & 2 & $13,296,695$ & $13,296,773$ & - & rs344472188 & Apical loop & G & 0.1364 \\
\hline ssc-miR-23a & 2 & $65,308,117$ & $65,308,186$ & + & rs333787816 & Precursor stem & C & 0.5398 \\
\hline ssc-miR-30d & 4 & $6,948,669$ & $6,948,747$ & + & rs340704946 & Precursor stem & G & 0.4762 \\
\hline ssc-miR-371 & 6 & $56,427,208$ & $56,427,285$ & - & rs320008166 & Precursor stem & C & 0.2955 \\
\hline ssc-miR-429 & 6 & $63,491,921$ & $63,492,001$ & + & rs323906663 & Precursor stem & A & 0.2955 \\
\hline ssc-miR-326 & 9 & $9,581,944$ & $9,582,034$ & - & rs319154814 & Apical loop & A & 0.5852 \\
\hline ssc-miR-34c & 9 & $39,280,278$ & $39,280,357$ & + & rs321151601 & Mature region & A & 0.0805 \\
\hline ssc-miR-378-2 & 12 & $36,947,443$ & $36,947,510$ & + & rs341950320 & Precursor stem & A & 0.1176 \\
\hline ssc-miR-15b & 13 & $100,083,172$ & $100,083,269$ & + & rs334680106 & Precursor stem & $\mathrm{T}$ & 0.2706 \\
\hline ssc-miR-486 & 17 & $10,758,818$ & $10,758,899$ & - & rs335924546 & Precursor stem & $\mathrm{T}$ & 0.3391 \\
\hline ssc-miR-335 & 18 & $18,341,568$ & $18,341,659$ & - & rs334590580 & Precursor stem & C & 0.1875 \\
\hline
\end{tabular}

a SSC: porcine chromosome

${ }^{b}$ Alt. allele: alternative allele of the genotyped SNP

${ }^{\mathrm{C}}$ Frequency: frequency of the alternative allele in the Lipgen population 
expression levels of mRNAs that fulfilled the following conditions: (1) the $3^{\prime} \mathrm{UTR}$ of the mRNA contains at least one $7 \mathrm{mer}-\mathrm{m} 8$ site complementary to the seed of the miRNA harboring the SNP to be tested (as predicted with the locate tool from SeqKit software [46]), and (2) the existence of experimentally-validated mRNA-miRNA interactions between the given mRNA and the tested miRNA have been confirmed in humans according to information provided in the DIANA-Tarbase v8 database [63]. The first condition was established because we were interested in focusing our analyses on miRNA-mRNA pairs for which a direct physical interaction (through complementarity between the seed of the miRNA and a target site in the $3^{\prime}$ UTR of the mRNA) is feasible. The second condition was imposed because there is extensive evidence that in silico prediction or miRNA binding sites can have high false positive rates [64]. We considered as valid miRNA-mRNA interactions those that have been experimentally validated in humans by using wet lab methods such as cross-linking, ligation and sequencing of hybrids (CLASH), photoactivatable ribonucleoside-enhanced and high-throughput sequencing of RNA isolated by crosslinking and immunoprecipitation (PARCLIP and HITS-CLIP) and luciferase assays [63].

It should be noted that the seeds of the porcine miRNAs harboring the 12 genotyped SNPs were completely conserved with regard to the seeds of the human orthologous miRNA genes (see Additional file 4: Figure S1). This feature further supports the extrapolation of miRNAmRNA interactions experimentally validated in humans [63] to pigs. For the sake of completeness, we also carried out a complementary association analysis between miRNA SNPs and the mRNA levels of the whole sets of genes expressed in the GM and liver tissues (i.e., without applying the two conditions mentioned above) but the results of such analyses should be interpreted with caution due to reasons stated above.

In association analyses with lipid traits or gene expression phenotypes, the alternative hypothesis $\mathrm{H}_{1}: \delta \neq 0$ was contrasted against the null hypothesis $\mathrm{H}_{0}: \delta=0$ with a likelihood ratio test. The statistical significance of the associations between miRNA SNPs and lipid and mRNA expression phenotypes was assessed with a false discovery rate (FDR) approach [65].

\section{Pathway enrichment analysis}

The lists of probes/genes that are significantly associated at the nominal level $(P$-value $<0.05)$ with miRNA SNPs genotypes $(\mathrm{N}=12)$ after considering the criteria for miRNA target inference (see "Methods" above) were used as inputs for pathway enrichment analyses. The ClueGO v2.5.0 plug-in application [66] within the Cytoscape 3.8.2 software [67] was used for determining enriched Reactome and KEGG pathways. A one-sided hypergeometric test of significance was applied for determining enriched terms and multiple testing correction was implemented with the FDR method [65].

\section{Confirmation of associations between ssc-miR-326 rs319154814 genotypes and gene expression data by $R T-q P C R$}

The hepatic mRNA levels of three of the mRNA transcripts among the most significantly associated with ssc-miR-326 rs319154814 (G/A) genotype were analyzed by reverse transcription-quantitative polymerase chain reaction (RT-qPCR). The $\beta$-actin (ACTB) and TATAbox binding protein $(T B P)$ genes were used as endogenous controls, as previously reported [68]. In brief, $100 \mathrm{mg}$ of liver tissue from 10 selected samples (5 from each $G G$ and $A A$ genotypes) were placed in $1 \mathrm{~mL}$ TRIzol reagent (Invitrogen Corp., Carlsbad, CA, USA) and subsequently homogenized using the Lysing Matrix D reagent (MP Biomedicals, Santa Ana, CA) in a Precellys 24 tissue homogenizer (Bertin Instruments, Rockville, MD). Total RNA isolation was performed following the protocol described by Rio et al. [69]. Reverse transcription was achieved with the High-Capacity cDNA Reverse Transcription Kit (Applied Biosystems, Foster City, CA) following the instructions of the manufacturer. We used $1 \mu \mathrm{g}$ of total RNA as a template in a final volume of $20 \mu \mathrm{L}$ and the synthesized cDNA was diluted to $1: 20$ in Milli-Q water. One pair of primers spanning exon-exon junctions was designed for each gene (see Additional file 5: Table S4) with the Primer Express software (Life Technologies Corporation). Real-time qPCR reactions contained $10 \mu \mathrm{L}$ of SYBR Select Master Mix, $300 \mathrm{nM}$ of each primer, and $5 \mu \mathrm{L}$ of 1:20 cDNA dilution, in a final volume of $20 \mu \mathrm{L}$. Reactions were run in an ABI PRISM 7900HT instrument (Applied Biosystems, Foster City, CA). The thermal cycle was set as follows: $2 \mathrm{~min}$ at $50^{\circ} \mathrm{C}$, one denaturing step at $95^{\circ} \mathrm{C}$ during $10 \mathrm{~min}$, followed by 40 cycles of $15 \mathrm{~s}$ at $95^{\circ} \mathrm{C}$ and $1 \mathrm{~min}$ at $60^{\circ} \mathrm{C}$. Moreover, a melting curve analysis was performed (i.e., $95^{\circ} \mathrm{C}$ for $15 \mathrm{~s}$, $60^{\circ} \mathrm{C}$ for $15 \mathrm{~s}$ and a gradual increase in temperature, with a ramp rate of $1 \%$ up to $95{ }^{\circ} \mathrm{C}$, followed by a final step of $95^{\circ} \mathrm{C}$ for $15 \mathrm{~s}$ ), in order to assess the specificity of the reactions. A standard curve with serial dilutions from a pool including all the cDNA samples was used to assess whether amplification efficiencies of the three genes under analysis were comprised in the 90 to $110 \%$ range. All reactions were run in triplicate.

The $2^{-\Delta \Delta \mathrm{Ct}}$ method [70] was used for the relative quantification of gene expression $(\mathrm{Rq})$, using the group of $G G$ samples as a calibrator. Subsequently, $\mathrm{Rq}$ values were $\log _{2}$ transformed and the significance of expression 
differences between $G G$ and $A A$ genotypes was assessed using the Welch's t-test for unpaired groups of samples [71] implemented in the t.test $\mathrm{R}$ function.

\section{Measurement of the expression of the ssc-miR-326 gene in pigs with different rs319154814 genotypes}

Liver samples from nine $A A$ and nine $G G$ pigs (rs319154814 genotype) were used to measure the expression of the ssc-miR-326 gene. Total RNA was isolated with TRIzol as previously described and reversetranscribed with the TaqMan Advanced MicroRNA Reverse Transcription Kit (Applied Biosystems, Foster City, CA), following the instructions of the manufacturer. Total RNA (10 ng) diluted in a volume of $2 \mu \mathrm{L}$ was used as a template to generate and pre-amplify cDNA by carrying out four consecutive reactions (poly-A tailing, adaptor ligation, reverse transcription and pre-amplification). The resulting pre-amplified cDNA was diluted to 1:50 in Milli- $Q$ water. To measure ssc-miR-326 expression levels, both let-7a and miR-26a-5p were chosen as endogenous controls following Timoneda et al. [72]. To conduct the experiments for the three miRNAs under investigation (one target and two controls), available inventoried TaqMan advanced miRNA assays (Applied Biosystems, Foster City, CA) for each miRNA were purchased. The reactions were performed in a final volume of $15 \mu \mathrm{L}$ that contained: $3.75 \mu \mathrm{L}$ of 1:50 cDNA, $3 \mu \mathrm{L}$ of nuclease-free water, $7.5 \mu \mathrm{L}$ of TaqMan Fast Advanced Master Mix $(2 \times)$, and $0.75 \mu \mathrm{L}$ TaqMan Advanced miRNA Assay $(20 \times)$. Each reaction was carried out in triplicate in a 384-well plate. Reactions were run in a QuantStudio 12K Flex Real-Time PCR System (Applied Biosystems, Foster City, $\mathrm{CA})$. The thermal cycle program was the same as that used for qPCR experiments on mRNA transcripts. No standard dissociation curve was performed. The results were analyzed applying the $2^{-\Delta \Delta C t}$ method [70] for the relative quantification of miRNA expression (Rq), using the group of GG samples as a calibrator. Subsequently, $\mathrm{Rq}$ values were $\log _{2}$ transformed and the significance of expression differences between $G G$ and $A A$ genotypes was assessed using a Welch's t-test for unpaired groups as used for mRNA qPCR analyses [71].

\section{Results}

\section{Differential segregation of SNPs mapping to microRNA} genes in pigs and wild boars from Europe and Asia

In total, 58,537,491 million SNPs were identified with the GATK haplotype caller tool [41] in a dataset comprising 120 WGS from 40 EDM, 40 ADM, 20 EWB and 20 AWB individuals (see Additional file 1: Table S1). The distribution of these SNPs within different annotated regions (i.e. protein coding genes, exons, introns, $3^{\prime}$ UTRs, miRNAs, IncRNAs, snRNAs, snoRNAs and pseudogenes) is available in Additional file 6: Table S5. Most of these SNPs were biallelic (96.82\%), 777,008 (1.33\%) were triallelic, 1,864,388 (3.18\%) had a deletion allele and 57,878 $(0.098 \%)$ showed four or more alleles (multi-allelic). From the set of multi-allelic SNPs, 1765 SNPs mapped to exons, and 531 of them were clustered in a set of 74 genes enriched in olfactory receptors (see Additional file 7: Table S6). This latter result is consistent with the potential presence of copy number variation $(\mathrm{CNV})$ in such locations [73]. Alternative allele frequencies were consistently high $(>0.5)$ for $9.25 \%$ of the variants, whereas low (between 0.05 and 0.01 ) and very low $(<0.01)$ alternative allele frequencies were detected in $27.85 \%$ and $19.62 \%$ of SNPs, respectively.

After filtering, 19,720,314 autosomal whole-genome SNPs were selected for assessing population structure based on PCA clustering techniques. The PCA showed a strong genetic differentiation among Asian and European populations (Fig. 2a). In contrast, domestic pigs and wild boars, and particularly those with a European origin, did not show such a strong divergence. Asian pigs and wild boars displayed a certain level of genetic differentiation and were more diverse than their European counterparts.

With regard to miRNA variability, the 370 porcine miRNA genes annotated in the manually curated miRCarta v1.1 database [44] were selected, and SNPs within these genes were retrieved. In total, 285 SNPs residing in 139 miRNAs (37.56\% of the total count) were identified (see Additional file 2: Table S2), which implies that the majority of miRNAs are monomorphic. Most of these 139 miRNAs (76.98\%) included one or two SNPs located within their predicted genomic boundaries, while $18.70 \%$ contained between three up to five SNPs, and $4.32 \%$ of them displayed more than seven SNPs (Additional file 4: Figure S2). Only 43 miRNA SNPs (15.09\%) were shared among the EDM, ADM, EWB and AWB populations (see Additional file 2: Table S2), and showed alternative alleles in at least one of the analyzed individuals in each group. The numbers of SNPs segregating in each of the four defined groups were 129 (EDM), 201 (ADM), 76 (EWB) and 172 (AWB), respectively (see Additional file 6: Table S5). With regard to precursor and mature regions, 41 and two SNPs were shared among the four populations under consideration, respectively (see Additional file 4: Figure S3A, B). None of the SNPs in the seed regions were shared by the four porcine populations (see Additional file 4: Figure S3C). Only three miRNA SNPs were found in the European dataset but not in the Asian dataset. In strong contrast, 55 miRNA SNPs were detected in the Asian dataset but not in the European dataset. Principal component analyses based on identified autosomal miRNA SNPs $(\mathrm{N}=260$, see Additional file 2: Table S2) showed poor differentiation between pigs and wild boars 

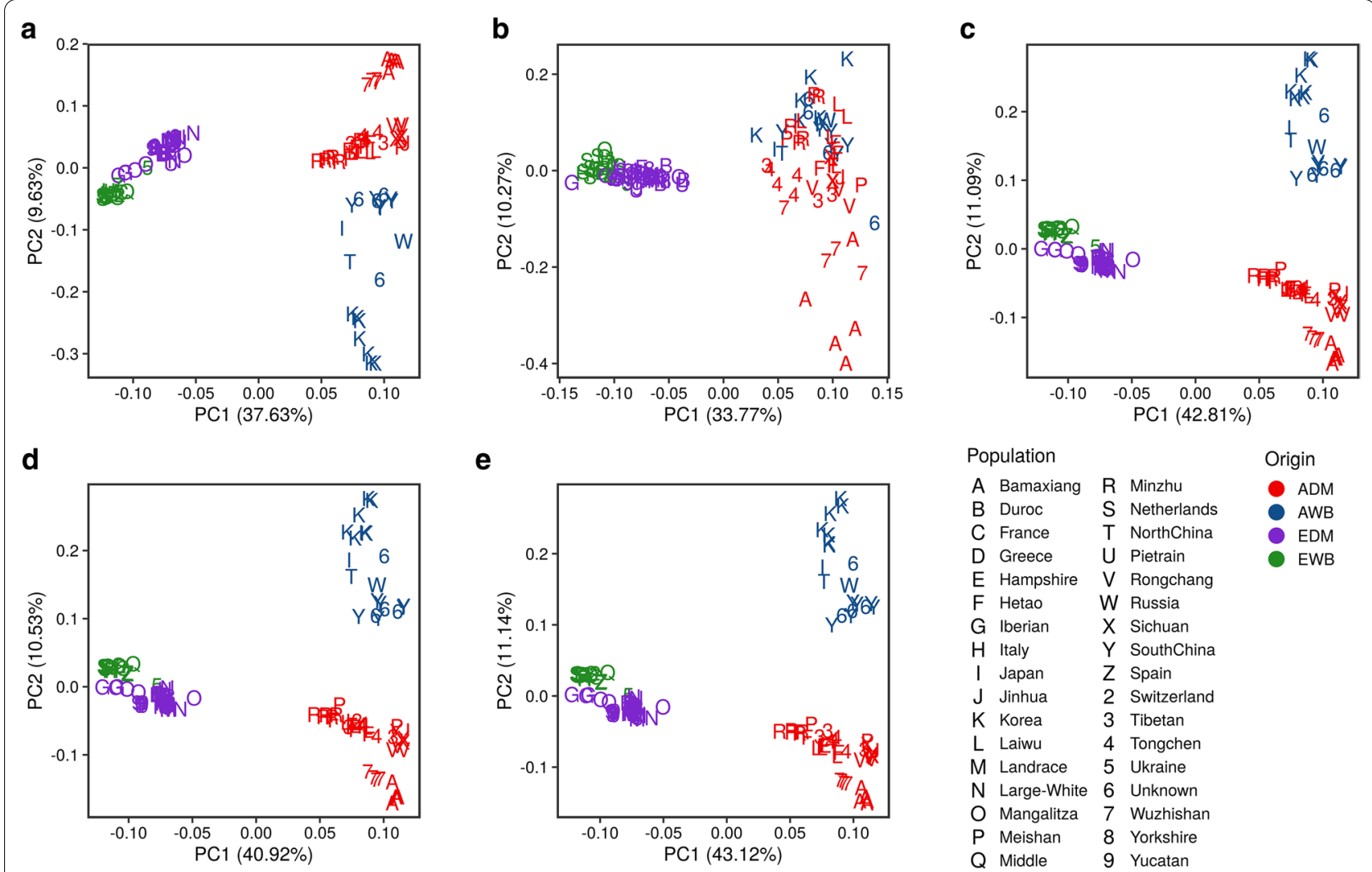

Fig. 2 Principal component analysis plots based on SNPs mapping to: a the whole-genome ( $N=19,720,314$ SNPs), b miRNA genes ( $N=285$ SNPs), c full 3'UTRs ( $N=709,343$ SNPs), d 3'UTR 7mer-m8 sites ( $N=107,196$ SNPs) and e 3'UTR 8mer sites ( $N=33,511$ SNPs), respectively. The symbol "Q" represents a wild boar sampled from the Middle East

(Fig. 2b), whereas the genetic divergence between European and Asian individuals was still apparent.

When we analyzed population structure based on whole-genome autosomal 3'UTR SNPs $(\mathrm{N}=709,343$ SNPs, Fig. 2c), 3'UTR 7mer-m8 site SNPs $(\mathrm{N}=107,196$ SNPs, Fig. 2d) and $3^{\prime}$ UTR 8mer site SNPs $(\mathrm{N}=33,511$ SNPs, Fig. 2e), the genetic differentiation between Asian vs. European populations was evident, in close concordance with results shown in Fig. 2a, b. However, we also detected a more pronounced differentiation between domestic pigs and wild boars, and this observation was particularly clear for Asian pigs and wild boars (Fig. 2c-e).

\section{Analysis of European and Asian populations shows reduced variability in porcine microRNAs}

About $47.76,57.36,44.77$ and $36.84 \%$ of the miRNA SNPs showed alternative allele frequencies $\leq 0.1$ in the ADM, EDM, AWB and EWB populations, respectively (Fig. 3 and see Additional file 2: Table S2). Variations located at mature miRNA and seed regions were enriched in rare or very rare variants compared to the variability of miRNA precursor regions (see Additional file 2: Table S2), with average alternative allele frequencies of $\sim 0.1$ for mature and seed miRNA polymorphisms. In contrast, the average frequency of the alternative allele observed for SNPs in precursor areas was $\sim 0.15$.

Moreover, the observed SNP density adjusted to $100 \mathrm{bp}$ for miRNA precursor, mature and seed regions consistently followed the order precursor $>$ mature $>$ seed when we considered the full set of 120 WGS. Indeed, one SNP per 100 bp was detected in precursor regions, whereas $\sim 0.86$ and $\sim 0.42$ SNPs per $100 \mathrm{bp}$ were observed in the mature and seed regions, respectively (Fig. 4a). After computing a non-parametric comparison of SNP density between the mature and seed regions with a Mann Whitney $\mathrm{U}$ test [50], a significant difference $(P$-value $=0.016)$ was detected. These results implied a 1.16 -fold reduction in SNP density between precursor and mature regions, whereas for seed regions, which are critical determinants of miRNA-mRNA interactions, the observed SNP density was $\sim 2.4$-fold lower than in precursor regions. This differential distribution 

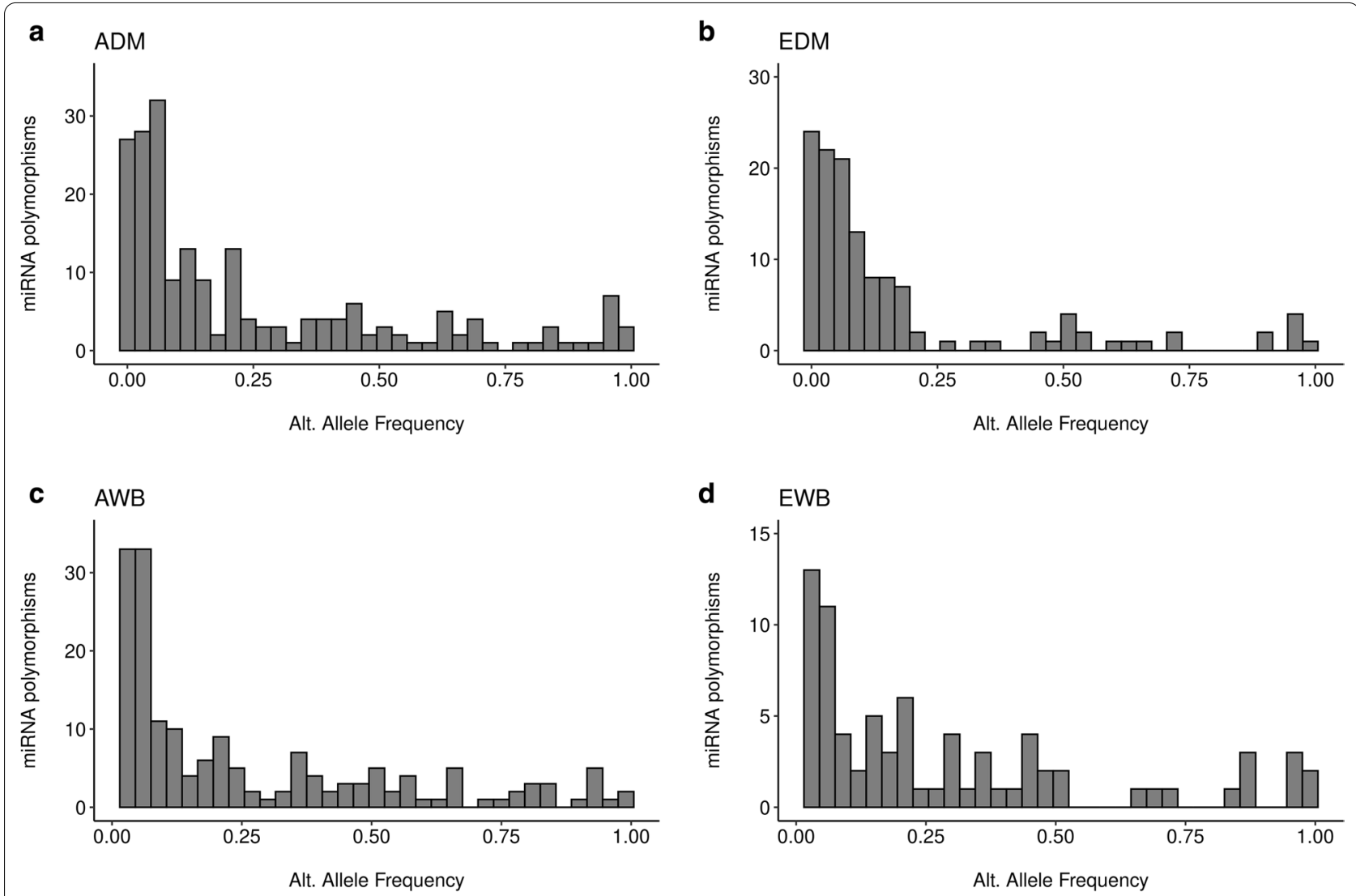

Fig. 3 Alternative allele frequency distribution of polymorphisms located at miRNA loci in a Asian domestic pigs (ADM), b European domestic (EDM) pigs, $\mathbf{c}$ Asian wild boars (AWB) and $\mathbf{d}$ European wild boars (EWB)

of the SNP density across miRNA regions (precursor $>$ mature $>$ seed) was also observed in each of the analyses performed in the ADM, EDM, AWB and EWB groups (Fig. 4a). With regard to variants located within mature miRNAs $(\mathrm{N}=64)$, both inside $(\mathrm{N}=12)$ and outside seed regions $(\mathrm{N}=52)$, their distribution along the sequence of the mature miRNA $(\sim 22 \mathrm{nt})$ showed a characteristic pattern (Fig. 4b): among all the detected SNPs, the first position of the mature miRNA $5^{\prime}$ end, which binds to the MID domain of the Argonaute protein in the miRISC complex, exhibited a SNP density of 0.49 SNPs per $100 \mathrm{bp}$. Such scarcity in polymorphic sites was also observed when considering the following positions 2 to 8 in the mature miRNA sequence (seed region), with an average of $\sim 0.42$ SNPs per $100 \mathrm{bp}$ across the whole seed and up to $\sim 0.73$ SNPs per $100 \mathrm{bp}$ at position 6 of the mature miRNA. In contrast, the interval between positions 9 to 12 (with no functional implications in terms of mRNA targeting) showed an increased average SNP density of $\sim 0.98$ SNPs per 100 bp. Positions 13 to 18 of the mature miRNA, which roughly correspond to a functional region providing additional anchor pairing to the seed region, showed a decreased SNP density, particularly at positions 16 and 17 (Fig. 4b). In addition, an increased SNP density was found at positions 19 to 22. Furthermore, the SNP density in miRNA genes $(\mathrm{N}=370$, Fig. $4 \mathrm{c})$ was $\sim 2.6$ fold lower ( $P$-value $<0.01$, see "Methods") than in their flanking regions $( \pm 1 \mathrm{~kb})$. A list of the miRNA SNPs $(\mathrm{N}=64)$ located at mature miRNAs and their genomic coordinates within the mature miRNA sequence is in Additional file 8: Table S7.

\section{Statistics of the whole-genome sequencing of five Duroc boars and association analyses}

As previously explained, we sequenced the genomes of five Duroc pigs which founded a population of 350 offspring with the goal of identifying SNPs in miRNA genes and investigating their association with mRNA expression and lipid phenotypes. Mean coverage values of the five pig genomes ranged from $37.67 \times$ to $46.6 \times$, with more than $98.6 \%$ of the genome covered by at least 10 reads in all five samples, and $96.71 \%$ of the genome covered by at least 15 reads. More details about coverage 

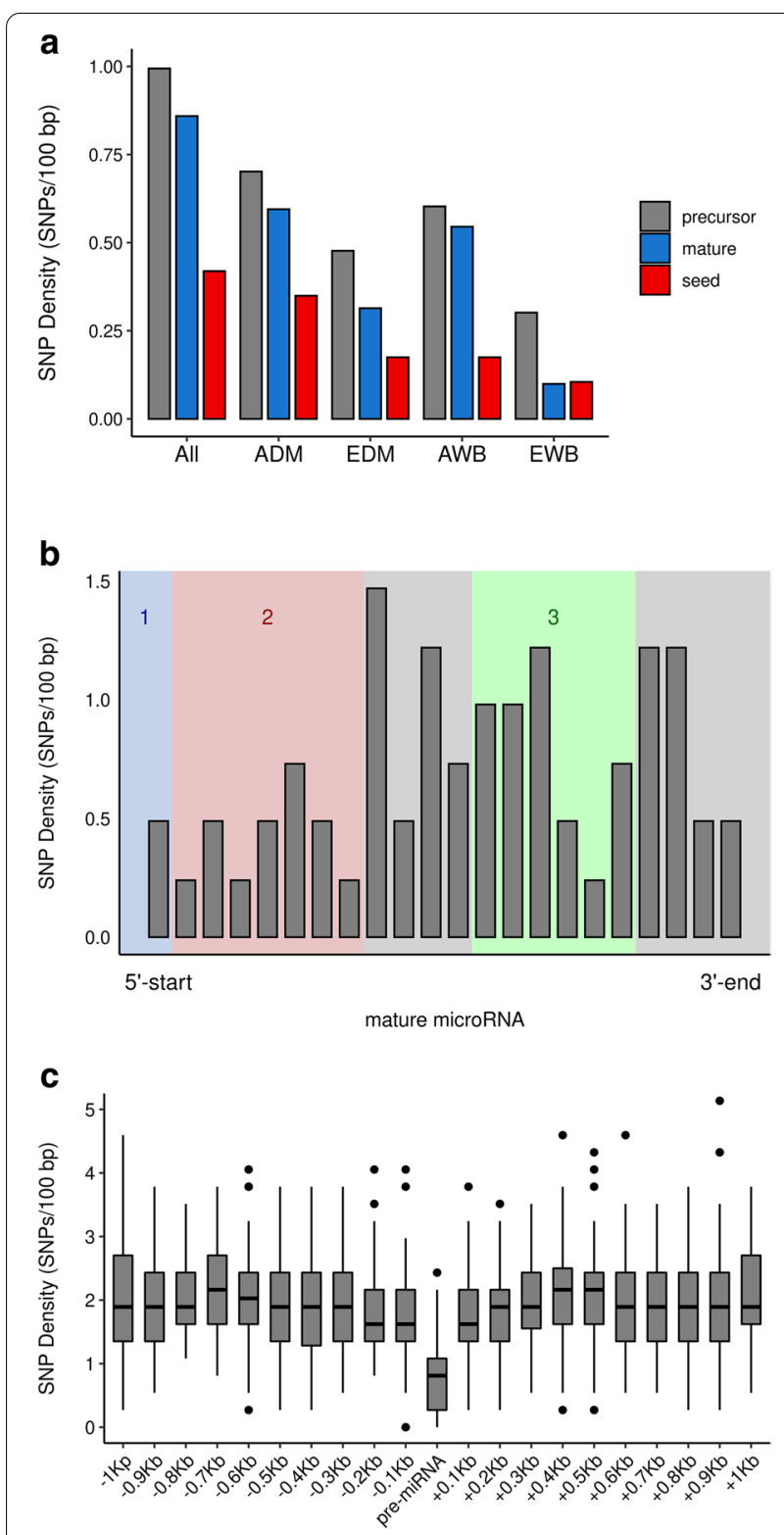

Fig. 4 a SNP density per 100 bp for each analyzed miRNA region considering the full set of 120 porcine whole-genome sequences (WGS), as well as each WGS from the ADM, EDM, AWB and EWB groups. $\mathbf{b}$ SNP density across mature miRNA regions. 1: anchor (1 st $5^{\prime}$ end position), 2: seed (positions 2 to 8 ) and 3: supplemental pairing (positions 13 to 18). c SNP density in miRNA loci and flanking regions considering a window of $\pm 1 \mathrm{~kb}$ divided in 10 upstream and downstream 100 bp bins

and genome mapping parameters are in Additional file 4: Figure S4 and Additional file 9: Table S8. After performing variant calling on mapped reads, $13,839,422$ SNPs passed the set quality criteria, whereas 3,721,589 InDels were detected. Moreover, 1,643,861 InDels (44.17\%) and 6,034,548 SNPs (43.60\%) were located in annotated protein coding loci. Fifty-five SNPs and five InDels were located in the 370 miRNA loci annotated in the Sscrofa.11.1 genome assembly according to the miRCarta database [44]. From these, 53 variants (96.36\%) were shared with the set of 285 miRNA SNPs found in the 120 WGS reported before (see Additional file 10: Table S9). After performing quality check of the variants mapping to miRNA genes, 49 SNPs and five InDels passed all set filtering criteria.

Among the 49 identified miRNA polymorphisms, we selected 12 SNPs on the basis of their location in relevant annotated miRNA loci (Table 1 and see Additional file 10: Table S9) for genotyping and performing association analyses. Microarray expression data comprised 23,998 probes, of which 12,131 (50.55\%) and 12,787 (53.28\%) corresponded to genes expressed in GM and liver tissues, respectively.

To provide a comprehensive view about the association between miRNA genotypes and liver and muscle mRNA levels, we carried out an association analysis between miRNA genotypes and the whole set of expressed probes/genes in these two tissues (Table 2). The results show that there are hundreds of associations at the nominal level, whereas the number of significant associations is strongly reduced after multiple testing correction. This analysis does not take into account the existence of complementarity between the seed of the miRNA and the porcine $3^{\prime} \mathrm{UTR}$ of target mRNAs or the functional validation data obtained in humans (see "Methods" section). A second analysis that took these two criteria into account revealed that from the set of 12 genotyped SNPs listed in Table 1, only two SNPs showed significant associations $(q$-value $<0.1)$ with the levels of mRNAs predicted to be miRNA targets (Table 3 and see Additional file 11: Table S10). Further details on the target sites at the $3^{\prime} \mathrm{UTR}$ of mRNA transcript expression profiles that are significantly associated with miRNA SNP genotypes (Table 3) are shown in Additional file 12: Table S11. All genes displaying significant associations $(q$-value $<0.1)$ in this second analysis ranked among the top $1 \%$ of significance (see Additional file 13: Table S12) in the condition-free analysis, except for transcripts associated with ssc-miR-326 genotypes in the liver, which ranked among the top 5\% (see Additional file 13: Table S12). We observed that for several of the significant associations between miRNA and mRNA pairs reported in Table 3 and see Additional file 11: Table S10, the interaction was classified as 7 -mer-m 8 by our targeting approach with the locate tool of SeqKit toolkit [46] when, in reality, it was of type 8 mer with one mismatch (see Additional file 12: Table S11). 
Table 2 Number of significant associations between the 12 genotyped miRNA SNPs and the whole set of mRNAs expressed in the gluteus medius (GM) skeletal muscle $(\mathrm{N}=89)$ and liver $(\mathrm{N}=87)$ tissue samples from Duroc pigs

\begin{tabular}{|c|c|c|c|c|c|}
\hline \multirow[t]{2}{*}{ SNP (chromosome position) } & \multirow[t]{2}{*}{ Type } & \multicolumn{4}{|c|}{ Significant associations } \\
\hline & & $\begin{array}{l}\mathrm{GM} \\
(P \text {-value }<0.05)\end{array}$ & $\begin{array}{l}\text { LIVER } \\
(P \text {-value }<0.05)\end{array}$ & $\begin{array}{l}\mathrm{GM} \\
(q \text {-value }<0.1)\end{array}$ & $\begin{array}{l}\text { LIVER } \\
(q \text {-value }<0.1)\end{array}$ \\
\hline rs81349391 (1:164,026,014 bp) & ssc-miR-339 apical loop (A/G) & 456 & 356 & 0 & 0 \\
\hline rs344472188 (2:13,296,736 bp) & ssc-miR-130a apical loop (A/G) & 320 & 1252 & 0 & 1 \\
\hline rs333787816 (2:65,308,181bp) & ssc-miR-23a precursor stem (T/C) & 2075 & 751 & 6 & 1 \\
\hline rs340704946 (4:6,948,743bp) & ssc-miR-30d precursor stem (A/G) & 563 & 398 & 0 & 0 \\
\hline rs320008166 (6:56,427,227 bp) & ssc-miR-371 precursor stem (T/C) & 270 & 365 & 2 & 8 \\
\hline rs323906663 (6:63,491,948 bp) & ssc-miR-429 precursor stem (G/A) & 328 & 406 & 2 & 8 \\
\hline rs319154814 (9:9,581,989bp) & ssc-miR-326 apical loop (G/A) & 372 & 2400 & 0 & 0 \\
\hline rs321151601 (9:39,280,312 bp) & ssc-miR-34c mature region (C/A) & 947 & 392 & 0 & 0 \\
\hline rs341950320 (12:36,947,510bp) & ssc-miR-378-2 precursor stem (G/A) & 792 & 322 & 0 & 0 \\
\hline rs334680106 (13:100,083,229 bp) & ssc-miR-15b precursor stem $(\mathrm{C} / \mathrm{T})$ & 277 & 205 & 0 & 0 \\
\hline rs335924546 (17:10,758,828 bp) & ssc-miR-486 precursor stem $(\mathrm{C} / \mathrm{T})$ & 1232 & 171 & 0 & 0 \\
\hline rs334590580 (18:18,341,582 bp) & ssc-miR-335 precursor stem (T/C) & 665 & 219 & 1 & 0 \\
\hline
\end{tabular}

This is a condition-free association analysis in which all expressed mRNAs are taken into account regardless of the existence of miRNA-mRNA complementarity and the availability of functional data validating miRNA-mRNA interactions in humans (see "Methods" for more information)

Table 3 Top significant associations after correction for multiple testing ( $q$-value $<0.1$ ) between miRNA SNPs and the mRNA expression of their potential targets in the gluteus medius (GM) skeletal muscle $(\mathrm{N}=89)$ and liver $(\mathrm{N}=87)$ tissue samples from Duroc pigs

\begin{tabular}{|c|c|c|c|c|c|c|c|c|c|}
\hline $\begin{array}{l}\text { SNP (chromosome } \\
\text { position) }\end{array}$ & Type & Tissue & Probe & ID & Gene & $\delta^{a}$ & $\mathrm{se}^{\mathrm{b}}$ & $P$-value & $q$-value ${ }^{c}$ \\
\hline \multirow{10}{*}{$\begin{array}{l}\text { rs333787816 } \\
\text { (2:65,308,181 bp) }\end{array}$} & \multirow{10}{*}{$\begin{array}{l}\text { ssc-miR-23a } \\
\text { precursor } \\
\text { stem (T/C) }\end{array}$} & \multirow[t]{10}{*}{ GM } & Ssc.1790.1.S1_at & ENSSSCG00000000019 & NUP50 & -0.1052 & 0.0288 & $3.849 \mathrm{E}-04$ & $6.157 \mathrm{E}-02$ \\
\hline & & & Ssc.12493.1.A1_at & ENSSSCG00000024027 & PAFAH1B2 & -0.2021 & 0.0565 & $3.921 \mathrm{E}-04$ & $6.157 \mathrm{E}-02$ \\
\hline & & & Ssc.13786.1.A1_at & ENSSSCG00000003857 & ZYG11B & 0.2614 & 0.0730 & $4.341 \mathrm{E}-04$ & 6.157E-02 \\
\hline & & & Ssc.8682.2.A1_at & ENSSSCG00000014240 & CSNK1G3 & -0.1532 & 0.0434 & $4.515 E-04$ & 6.157E-02 \\
\hline & & & Ssc.23976.1.S1_at & ENSSSCG00000012252 & DDX3X & -0.4318 & 0.1217 & $5.095 \mathrm{E}-04$ & 6.157E-02 \\
\hline & & & Ssc.4948.1.S1_at & ENSSSCG00000034725 & UBE2R2 & 0.1726 & 0.0493 & $5.906 \mathrm{E}-04$ & 6.157E-02 \\
\hline & & & Ssc.21303.1.S1_at & ENSSSCG00000003630 & AGO1 & 0.1016 & 0.0275 & $6.775 E-04$ & $6.157 \mathrm{E}-02$ \\
\hline & & & Ssc.24035.2.A1_at & ENSSSCG00000005935 & AGO2 & 0.2896 & 0.0864 & $8.339 E-04$ & 6.157E-02 \\
\hline & & & Ssc.3706.1.S2_at & ENSSSCG00000040317 & SOD2 & -0.2987 & 0.0896 & $8.880 \mathrm{E}-04$ & 6.157E-02 \\
\hline & & & Ssc.3706.1.S1_at & ENSSSCG00000040317 & SOD2 & -0.4224 & 0.1261 & $8.884 \mathrm{E}-04$ & $6.157 \mathrm{E}-02$ \\
\hline \multirow{8}{*}{$\begin{array}{l}\text { rs319154814 } \\
\text { (9:9,581,989 bp) }\end{array}$} & \multirow{8}{*}{$\begin{array}{l}\text { ssc-miR-326 } \\
\text { apical loop } \\
\text { (G/A) }\end{array}$} & \multirow[t]{8}{*}{ LIVER } & Ssc.9544.2.S1_a_at & ENSSSCG00000016101 & CFLAR & 0.3246 & 0.0997 & $1.264 \mathrm{E}-03$ & $5.785 \mathrm{E}-02$ \\
\hline & & & Ssc.11661.2.S1_at & ENSSSCG00000009828 & PPPICC & 0.4508 & 0.1436 & $1.784 \mathrm{E}-03$ & $5.785 \mathrm{E}-02$ \\
\hline & & & Ssc.31172.3.S1_at & ENSSSCG00000008601 & SDC1 & 0.2507 & 0.0808 & $2.486 \mathrm{E}-03$ & $5.785 \mathrm{E}-02$ \\
\hline & & & Ssc.11044.1.A1_at & ENSSSCG00000003643 & SF3A3 & 0.1808 & 0.0561 & $2.598 \mathrm{E}-03$ & $5.785 \mathrm{E}-02$ \\
\hline & & & Ssc.23242.1.A1_at & ENSSSCG00000039426 & FSTL1 & 0.2407 & 0.078 & $2.606 \mathrm{E}-03$ & $5.785 \mathrm{E}-02$ \\
\hline & & & Ssc.12222.1.S1_at & ENSSSCG00000013233 & CELF1 & -0.1714 & 0.0574 & $3.210 E-03$ & 5.939E-02 \\
\hline & & & Ssc.10946.2.A1_at & ENSSSCG00000011917 & NAA50 & 0.2853 & 0.0993 & $4.070 E-03$ & $6.454 \mathrm{E}-02$ \\
\hline & & & Ssc.4516.2.S1_at & ENSSSCG00000013592 & ELAVL1 & 0.1666 & 0.0598 & $6.226 \mathrm{E}-03$ & 8.639E-02 \\
\hline
\end{tabular}

In this analysis, we consider only the expression of mRNAs with 3'UTR sites complementary to the seeds of the investigated miRNAs and with such interactions having been functionally validated in humans (see "Methods" for more information)

a $\delta$ : estimated allele substitution effect

${ }^{b}$ se: standard error of the substitution effect

c $q$-value: $q$-value calculated with the false discovery rate (FDR) approach [65] 
Two SNPs in the apical loop of ssc-miR-326 and in the precursor region of ssc-miR-23a are associated with the mRNA expression of several of their putative targets

When we analyzed the association between the rs319154814 (G/A) polymorphism located in the apical loop of ssc-miR-326 and gene expression data (Table 3), significant results were obtained after multiple testing correction $(q$-value $<0.1)$. More specifically, we detected eight significant associations between this SNP and the hepatic mRNA expression of experimentally confirmed targets of this miRNA (Table 3). For instance, the hepatic mRNA levels of the genes cellular FLICE-like inhibitory protein (CFLAR), protein phosphatase 1 catalytic subunit $\gamma$ (PPP1CC), syndecan 1 (SDC1), splicing factor $3 A$ subunit 3 (SF3A3) and follistatin-like 1 (FSTL1) were among the most significantly associated with ssc-miR-326 genotypes (Table 3). The expression levels of seven of these mRNAs associated with ssc-miR-326 rs319154814 $(G / A)$ genotypes (Table 3 ) were reduced in pigs that are homozygous for the mutated allele $(A A, \mathrm{~N}=32)$, as depicted in Fig. 5.

The mRNA levels of the PPP1CC, CFLAR and SF3A3 mRNA transcripts were further measured by RT-qPCR in 10 liver samples ( 5 belonging to each $A A$ or $G G$ rs319154814 genotypes). After comparing the mean expression for each of the three analyzed mRNA transcripts in both $A A$ and $G G$ genotype groups, only the $P P P 1 C C$ transcripts showed a significantly reduced expression in $A A$ pigs compared with their GG counterparts $(P$-value $=0.027$, Fig. 6a). The other two transcripts (CFLAR and SF3A3) also showed a reduced expression in pigs with genotype $A A$ (Fig. 6a), but the results were not significant. We further investigated whether the ssc-miR-326 rs319154814 (G/A) genotypes were associated with the levels of this very same miRNA in the liver by using a specific Taqman Advanced miRNA probe assay. In this qPCR analysis, pigs with $A A$ genotypes for the rs319154814 polymorphism showed an increased $\sim 1$.9-fold expression of ssc-miR-326 transcripts compared with their GG counterparts, although this difference did not reach statistical significance $(P$-value $=0.178$, Fig. $6 b)$.

The rs333787816 $(T / C)$ polymorphism, located in the precursor region, immediately downstream of the mature ssc-miR-23a sequence, was also significantly associated with 36 experimentally confirmed targeted genes in the GM muscle tissue ( $q$-value $<0.1$, Table 3 , and see Additional file 11: Table S10). From these, it is worth mentioning the Argonaute RISC component 1
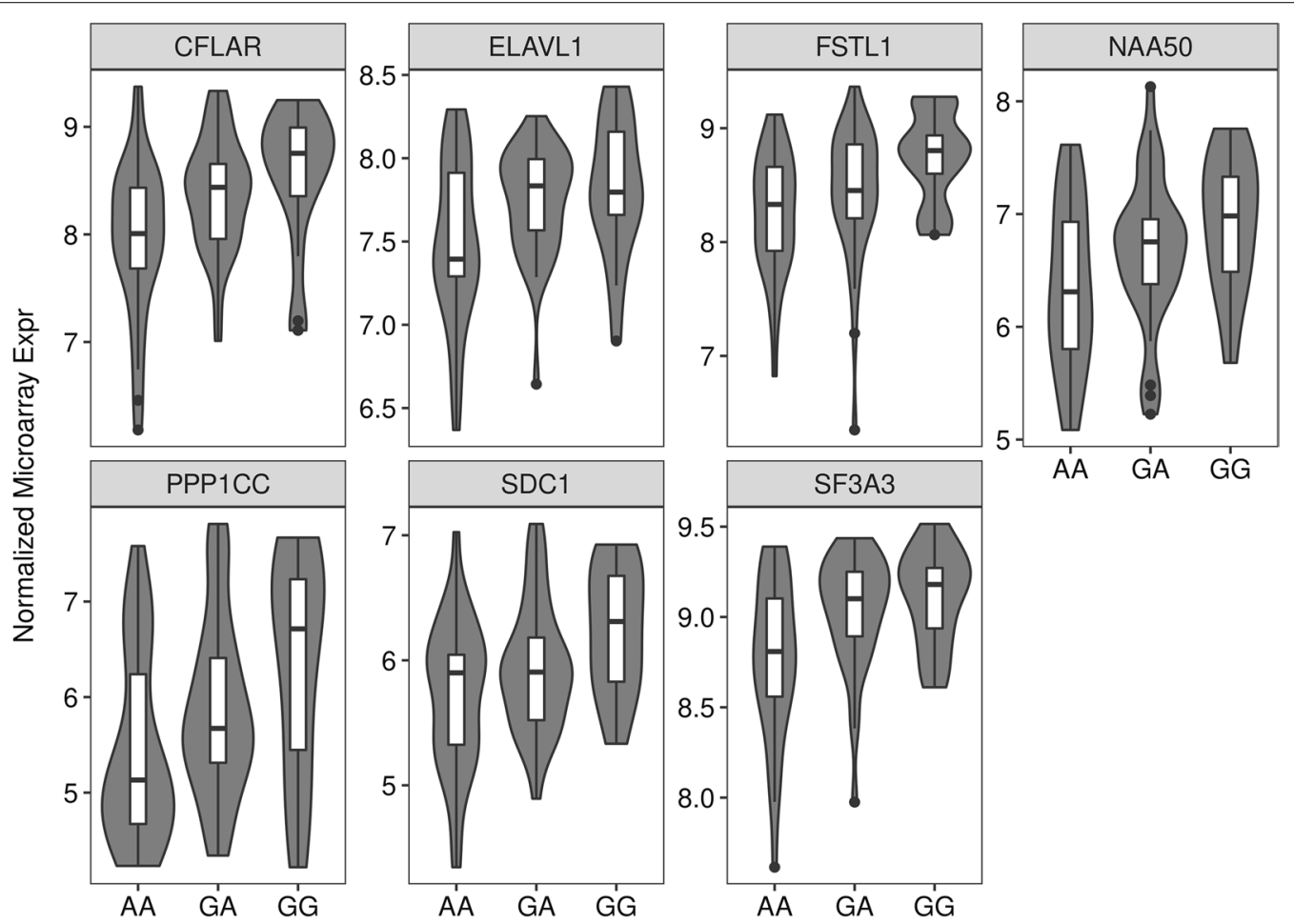

Fig. 5 Hepatic mRNA expression levels of the CFLAR, PPP1CC, SDC1, SF3A3, FSTL1, NAA50 and ELAVL1 genes according to the genotype of the rs319154814 (G/A) apical loop SNP in the ssc-miR-326 gene. The number of individuals representing each genotype were: $G G(N=17), G A(N=37)$ and $A A(N=32)$ 

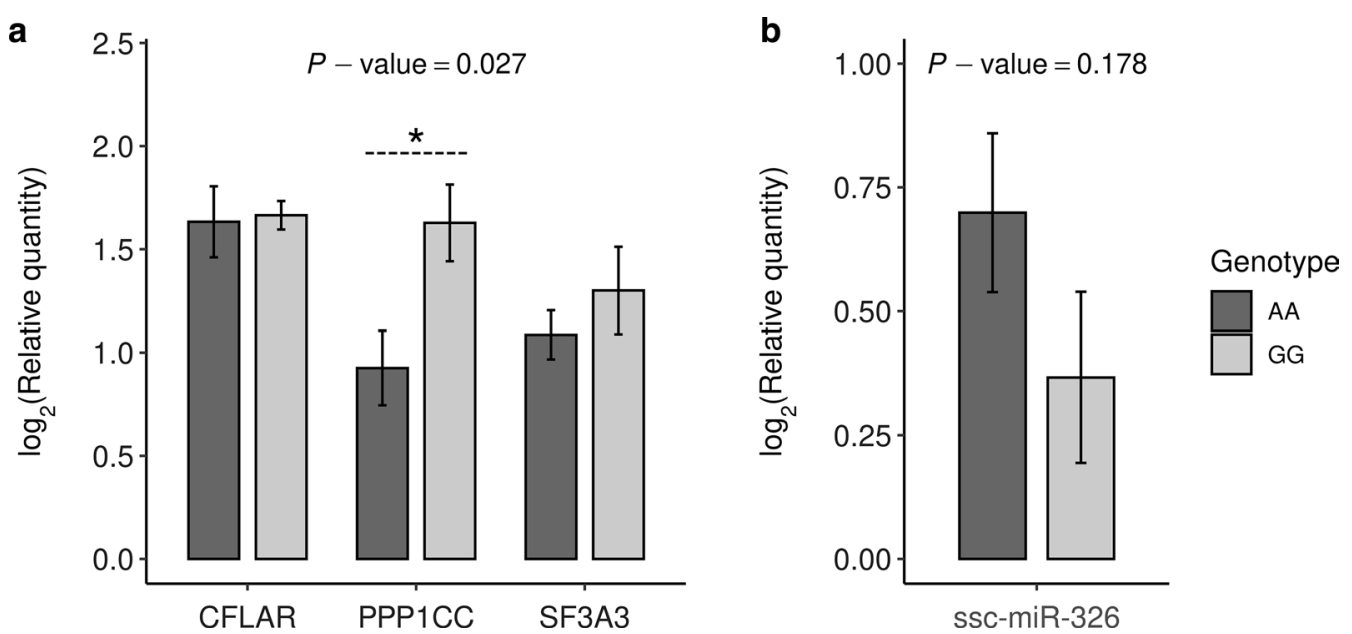

Fig. 6 a Barplots depicting qPCR $\log _{2}$ transformed relative quantities (Rq) for CFLAR, PPP1CC and SF3A3 mRNA transcripts measured in the liver tissue. The number of individuals representing each genotype were: $G G(N=5)$ and $A A(N=5)$. b Barplots depicting qPCR $\log _{2}$ transformed relative quantities (Rq) for ssc-miR-326 transcripts measured in the liver tissue. The number of individuals representing each genotype were: $G G(N=9)$ and $A A(N=9)$

(AGO1) and the Argonaute RISC catalytic component 2 (AGO2) mRNAs. Both $A G O 1$ and $A G O 2$ genes showed lowered mRNA expression levels in homozygous $C C$ pigs with respect to their $T T$ and $T C$ counterparts (data not shown).

\section{Pathway enrichment analyses of significantly associated mRNA targets provide insights of putative metabolic functions of miRNAs}

After performing pathway enrichment analyses on the sets of putative targeted mRNA transcripts that are significantly associated with miRNA SNP genotypes $(P$-value $<0.05)$, several biological categories were identified and reported in Additional file 14: Table S13. To mention a few of the most relevant findings, the sscmiR-23a genotype was associated with several regulatory pathways (post-transcriptional silencing by small RNAs, competing endogenous RNAs regulate PTEN translation, regulation of PTEN mRNA translation, regulation of RUNX1 expression and activity, etc.) involving genes such as $A G O 1, A G O 2$ or TNRC6C, which play relevant roles in miRNA-mediated gene regulation [74]. Among the sets of mRNAs nominally associated with the ssc-miR-30d genotype in GM, we identified the regulation of ornithine decarboxylase activity or cellular response to hypoxia, as well as mature mRNA transport after splicing (see Additional file 14: Table S13).

In the liver tissue, the ssc-miR-326 genotype was significantly associated with the expression of several circadian clock regulatory transcripts e.g., histone deacetylase 3 (HDAC3), period 1 (PER1) and the already mentioned $P P P 1 C C$ gene. Moreover, negative regulation of DDX58/IFIH1 signaling of type I interferon response and p53 signaling were pathways enriched in the sets of mRNAs nominally associated with ssc-miR130a genotypes (see Additional file 14: Table S13).

\section{Porcine lipid phenotypes are associated with the genotypes of miRNA genes}

We also evaluated the association between miRNA SNPs and several lipid-related phenotypes recorded in the Lipgen population (see Additional file 3: Table S3). Only the rs319154814 $(G / A)$ variant in the ssc-miR-326 gene was significantly associated $(q$-value $<0.1)$ with lipid traits (Table 4 and see Additional file 15: Table S14). More specifically, we found significant associations with myristic acid (C14:0) content in both LD and GM muscles, as well as with gadoleic acid (C20:1) content and the ratio between PUFA and MUFA in the LD muscle (Table 4 and see Additional file 15: Table S14). We also observed several additional associations between the rs319154814 (G/A) SNP and fatty acid composition traits, but they were significant only at the nominal level $(P$-value $<0.01$, Table 4$)$. Other apical loop SNPs such as rs81349391 $(A / G)$ at ssc-miR-339 and rs344472188 $(T / C)$ at ssc-miR-130a were significantly associated at the nominal level $(P$-value $<0.01)$ with palmitic acid (C16:0) content and SFA and UFA proportion in the GM muscle, as well as with backfat thickness. As shown in Table 4, a SNP located in the 
Table 4 Significant associations at the nominal level $(P$-value $<0.01)$ and after multiple testing correction $(q$-value $<0.1$; in italics) between 12 genotyped miRNA SNPs and lipid phenotypes recorded in a population of Duroc pigs $(\mathrm{N}=345)$

\begin{tabular}{|c|c|c|c|c|c|c|}
\hline SNP (chromosome position) & Type & Trait & $\delta$ & se & $P$-value & $q$-value \\
\hline \multirow[t]{3}{*}{ rs81349391 (1:164,026,014bp) } & \multirow[t]{3}{*}{ ssc-miR-339 apical loop (A/G) } & GM (C16:0) & -0.3234 & 0.1396 & $2.005 E-02$ & $3.772 \mathrm{E}-01$ \\
\hline & & GM SFA & -0.4394 & 0.2030 & $2.969 \mathrm{E}-02$ & $3.772 \mathrm{E}-01$ \\
\hline & & GM UFA & 0.4392 & 0.2030 & $2.978 \mathrm{E}-02$ & $3.772 \mathrm{E}-01$ \\
\hline rs344472188 (2:13,296,736 bp) & ssc-miR-130a apical loop (T/C) & BFTLR & -1.2084 & 0.5914 & 4.087E-02 & $9.683 \mathrm{E}-01$ \\
\hline rs340704946 (4:6,948,743bp) & ssc-miR-30d precursor stem (A/G) & GM (C20:0) & -0.0219 & 0.0098 & $2.541 \mathrm{E}-02$ & $5.779 \mathrm{E}-01$ \\
\hline rs320008166 (6:56,427,227 bp) & ssc-miR-371 precursor stem (T/C) & GM IMF & 0.5368 & 0.2461 & $3.104 \mathrm{E}-02$ & $9.060 \mathrm{E}-01$ \\
\hline rs323906663 (6:63,491,948 bp) & ssc-miR-429 precursor stem (G/A) & GM IMF & 0.5682 & 0.2482 & $2.348 \mathrm{E}-02$ & 8.923E-01 \\
\hline \multirow[t]{10}{*}{ rs319154814 (9:9,581,989bp) } & \multirow[t]{10}{*}{ ssc-miR-326 apical loop (G/A) } & $L D(C 14: 0)$ & 0.0885 & 0.0213 & $8.722 E-05$ & $3.314 E-03$ \\
\hline & & GM (C14:0) & 0.0700 & 0.0184 & $3.942 E-04$ & $7.489 E-03$ \\
\hline & & LDPUFAMUFA & -0.0506 & 0.0188 & $7.109 E-03$ & $7.653 E-02$ \\
\hline & & $L D(C 20: 1)$ & 0.0349 & 0.0125 & $8.055 E-03$ & $7.653 E-02$ \\
\hline & & LD (C20:4) & -0.2918 & 0.1268 & $2.092 \mathrm{E}-02$ & $1.438 \mathrm{E}-01$ \\
\hline & & LD MUFA & 0.9512 & 0.3971 & $2.485 \mathrm{E}-02$ & $1.438 \mathrm{E}-01$ \\
\hline & & $\operatorname{LD}(C 18: 1)$ & 0.8349 & 0.3624 & $2.814 \mathrm{E}-02$ & $1.438 \mathrm{E}-01$ \\
\hline & & LD PUFA & -1.0670 & 0.5004 & $3.222 \mathrm{E}-02$ & $1.438 \mathrm{E}-01$ \\
\hline & & LD (C18:2) & -0.7266 & 0.3443 & $3.405 E-02$ & $1.438 \mathrm{E}-01$ \\
\hline & & GM (C18:0) & -0.2245 & 0.1115 & 4.423E-02 & $1.681 \mathrm{E}-01$ \\
\hline rs321151601 (9:39,280,312bp) & ssc-miR-34c mature region (C/A) & LD (C20:0) & -0.0262 & 0.0119 & $2.655 \mathrm{E}-02$ & $8.171 \mathrm{E}-01$ \\
\hline \multirow[t]{3}{*}{ rs335924546 (17:10,758,828bp) } & \multirow[t]{3}{*}{ ssc-miR-486 precursor stem $(\mathrm{C} / \mathrm{T})$} & GM (C20:4) & 0.3396 & 0.1465 & $2.100 \mathrm{E}-02$ & 4.555E-01 \\
\hline & & LD (C14:0) & -0.0586 & 0.0256 & $2.937 \mathrm{E}-02$ & $4.555 \mathrm{E}-01$ \\
\hline & & LD IMF & 0.3300 & 0.1553 & $4.748 \mathrm{E}-02$ & $4.555 \mathrm{E}-01$ \\
\hline \multirow[t]{4}{*}{ rs334590580 (18:18,341,582 bp) } & \multirow[t]{4}{*}{ ssc-miR-335 precursor stem (T/C) } & GM (C16:0) & -0.4305 & 0.1558 & $5.862 \mathrm{E}-03$ & $1.201 \mathrm{E}-01$ \\
\hline & & GM (C20:0) & 0.0331 & 0.0121 & $6.321 \mathrm{E}-03$ & $1.201 \mathrm{E}-01$ \\
\hline & & GM UFA & 0.5231 & 0.2278 & $2.147 \mathrm{E}-02$ & $2.045 \mathrm{E}-01$ \\
\hline & & GM SFA & -0.5229 & 0.2278 & $2.153 \mathrm{E}-02$ & $2.045 E-01$ \\
\hline
\end{tabular}

GM: gluteus medius skeletal muscle; LD: longissimus dorsi skeletal muscle; BFTLR: backfat thickness at the last rib; IMF: intramuscular fat content; SFA: saturated fatty acids content; UFA: unsaturated fatty acids content; PUFA: polyunsaturated fatty acids content; MUFA: monounsaturated fatty acids content; C14:0: myristic content; C16:0: palmitic content; C18:0: stearic content; C18:1: oleic content; C18:2: linoleic content; C20:0 arachidic content; C20:1: gadoleic content; C20:4: arachidonic content

a $\delta$ : estimated allele substitution effect

b se: standard error of the substitution effect

c $q$-value: $q$-value calculated with the false discovery rate (FDR) approach [65]

precursor $3^{\prime}$ stem of ssc-miR-30d showed a nominally significant association with arachidic acid (C20:0) content. Other significant associations at the nominal level were, for instance, those between the rs334590580 $(T / C)$ SNP located at the precursor stem region of sscmiR-335 and palmitic and arachidic acids content in GM tissue (Table 4).

\section{Discussion}

Divergent patterns of variation for microRNA and 3'UTR polymorphisms in Asian and European pigs and wild boars The PCA revealed the existence of a detectable genetic differentiation between Asian and European populations, with the latter showing reduced levels of diversity compared to the former. Groenen et al. [75] investigated the variability of pig genomes and found that
Asian pigs and wild boars are more diverse than their European counterparts and that both gene pools split during the mid-Pleistocene $\sim 1.6$ to 0.8 Mya. Calabrian glacial intervals probably favored a restricted gene flow between these two gene pools [75]. The high variability of Asian populations could be explained by the fact that Sus scrofa emerged as a species in Southeast Asia ( 5.3 to $3.5 \mathrm{Mya}$ ) and then dispersed westwards until reaching Europe $\sim 0.8 \mathrm{Mya}$ [76]. This initial founder effect, combined with the occurrence of strong bottlenecks, reduced the genetic diversity of European wild boars [75].

While genetic differentiation between wild boar and pig populations was clearly detected when the wholegenome SNP dataset was considered (Fig. 2a), this was less evident in the PCA based on miRNA SNPs (Fig. 2b), probably because the small number $(\mathrm{N}=285$ 
SNPs) of markers used in this analysis limited the resolution with which population differentiation could be detected. We also found that the degree of population differentiation between Asian domestic pigs and Asian wild boars increased when PCA are built on the basis of SNPs located in the whole $3^{\prime} \mathrm{UTRs}(\mathrm{N}=709,343 \mathrm{SNPs})$, 3'UTR 7mer-m8 sites ( $\mathrm{N}=107,196 \mathrm{SNPs})$ or 3'UTR 8mer $(\mathrm{N}=33,511$ SNPs) sites (Fig. 2c-e). Potential effects of $3^{\prime}$ UTR SNPs are: modulation of mRNA expression or changes in secondary structure, stability, localization, translation, and binding to miRNAs and RNA-binding proteins [77]. Purifying selection is less intense in $3^{\prime}$ UTRs than in protein-coding regions, which implies that $3^{\prime}$ UTRs evolve faster and accumulate a larger fraction of recent polymorphisms contributing to population differentiation [78].

\section{Low SNP density in microRNA genes and lack of uniform SNP distribution across sites}

We have found that, in general, miRNA loci have a substantially lower SNP density in their seeds compared with mature and precursor regions (Fig. 4a). When we analyzed the SNP density in miRNA loci and their flanking regions $( \pm 1 \mathrm{~kb})$, a significantly reduced number of SNPs per $100 \mathrm{bp}$ was found in miRNAs compared with upstream and downstream flanking sequences (Fig. 4c). These results are in agreement with data reported by Omariba et al. [79] and Saunders et al. [5].

The low variability of miRNA genes, a feature that is particularly evident in the seed region, is probably due to the intense effects of purifying selection. Indeed, the importance of the miRNA seeds is revealed by the high conservation of their sequence across species $[16,23$, 48], as this sequence ultimately determines the success of miRNA-mRNA interactions [2]. In our study, 221, 52 and 12 SNPs were found in precursor, mature and seed regions within miRNA loci, respectively (see Additional file 2: Table S2 and Additional file 4: Figure S3). Gong et al. [80] described the existence of $40 \%$ polymorphic miRNAs in the human genome but only $16 \%$ of them displayed more than one SNP. In a more recent study, He et al. [81] reported 1879 SNPs in 1226 (43.6\%) human miRNA seed regions, and $97.5 \%$ of these polymorphisms had frequencies lower than $5 \%$. These results agree well with the overall distribution of the frequency of miRNA SNPs in the European and Asian populations analyzed in the current work (Fig. 3). He et al. [81] also demonstrated that $1587,749,340,102,31$, and 4 miRNAs harbored zero, one, two, three, four, and five SNPs, respectively, in their seed regions, which reflects that mutations in this critical functional region are not well tolerated. This distribution is similar to the one that we have observed in domestic pigs and wild boars, with 81,31, 11, 9 and 5 miRNAs harboring one, two, three, four and five SNPs (see Additional file 4: Figure S2). Only four and two miRNAs showed a total of seven and ten polymorphisms within their sequences (see Additional file 2: Table S2).

We also detected a non-uniform SNP density along the sequence of mature miRNAs (Fig. $4 \mathrm{~b}$ ). Gong et al. [80] showed that SNPs tend to concentrate in the middle region of the mature miRNA gene rather than at its $5^{\prime}$ and $3^{\prime}$ ends. Moreover, the same authors described an increased SNP density at positions 9 and 15 of the mature miRNA, a result that agrees well with ours (Fig. 4b). However, we have also identified an elevated number of polymorphic sites at positions 11, 19 and 20, a finding that does not match the human data presented by Gong et al. [80].

Several of the sites that show a reduced variability in porcine miRNAs exert critical functions (Fig. $4 \mathrm{~b}$ and see Additional file 8: Table S7). For instance, the first nucleotide of mature miRNAs plays an important role in the loading of the mature miRNA on the Argonaute protein to form the miRISC complex [82]. The second to eighth nucleotides in the mature miRNA correspond to the seed, where we found a consistently reduced SNP density (Fig. 4a, b) compared with other miRNA regions. This result was expected because this region has a crucial role in modulating the interaction between the mature miRNA and its $3^{\prime} \mathrm{UTR}$ binding sites. Polymorphisms in the seed region have the potential to disrupt the proper miRNA-mRNA pairing and thus alter biologically relevant regulatory pathways, which tend to be evolutionarily conserved [48]. Hence, these variants located in the miRNA seeds (see Additional file 8: Table S7) might favor the emergence of novel miRNA-mRNA interactions as well as the abolishment of conserved ones, thus modifying gene regulatory networks.

In contrast with the first eight $5^{\prime}$ positions of the miRNA, we found a higher SNP density at positions 9 to 12, which do not contribute substantially to miRNA target recognition [2] (Fig. 4b). Positions 13 to 16 facilitate $3^{\prime}$-compensatory pairing between the mature miRNA and targeted 3'UTRs [83], although only at marginal levels [84]. Nevertheless, in our porcine dataset only positions 16 and 17 showed a reduced SNP density (Fig. 4b). 


\section{Polymorphisms in microRNA genes show associations with the mRNA expression of several of their predicted targets}

We investigated the association of 12 genotyped SNPs mapping to miRNA genes and hepatic and muscle mRNA expression in Duroc pigs. Several hundreds of significant associations at the nominal level $(P$-value $<0.05)$ were detected in both GM muscle and liver tissues when the whole set of microarray expression profiles of mRNA transcripts were considered (Table 2). One the one hand, one pitfall of this analysis is that it does not take into account whether there is molecular evidence supporting the existence of direct miRNA-mRNA interactions displaying significant associations. On the other hand, indirect interactions between miRNAs and mRNAs might also exist i.e., a SNP regulating the expression of a miRNA might repress the translation of a given mRNA through a direct interaction (an event that would not necessarily imply any transcriptional decay, hence being undetectable in our experimental system) and, in turn, this might indirectly affect the expression of other multiple mRNAs regulated by the translationally repressed mRNA. In spite of this consideration, we decided to restrict our association analyses to miRNA-mRNA pairs with molecular evidence of potential interactions because we believe that with this stringent approach, we are able to minimize the chances of detecting spurious false positive results. The genomic positions of miRNA-binding sequences in the $3^{\prime}$ UTR of targeted mRNAs showing significant associations ( $q$-value $<0.1$, Table 3$)$ with miRNA genotypes are in Additional file 12: Table S11.

After correction for multiple testing ( $q$-value $<0.1$, Table 3), only SNPs mapping to the ssc-miR-23a and sscmiR-326 genes displayed significant associations with the expression levels of some of their putative mRNA targets. More in detail, the rs333787816 (T/C) polymorphism in the ssc-miR-23a gene showed significant associations with several putative target mRNAs, among which are $A G O 1$ and $A G O 2$ transcripts, two essential components of the miRNA-mediated silencing machinery $[85,86]$. The rs319154814 (G/A) polymorphism in the apical loop region of ssc-miR-326 also showed a significant association, after multiple testing correction, with the hepatic mRNA expression of several of its predicted and experimentally-confirmed targets (Table 3). In contrast, no association with GM muscle mRNA expression was observed. Tissue-specific differences in the expression of the miRNA or its mRNA targets might explain such an outcome. Indeed, the analysis of the distribution of miRNA expression across human tissues has shown that only a minority of miRNAs are expressed ubiquitously [87]. The hepatic mRNA targets showing the most significant association with rs319154814 (G/A)
ssc-miR-326 genotype were CFLAR, PPP1CC, SDC1, SF3A3 and FSTL1. The protein encoded by the PPP1CC gene belongs to the protein phosphatase PP1 subfamily, which is a ubiquitous serine/threonine phosphatase involved in regulating multiple cellular processes through dephosphorylation signaling. Among these processes, it is worth mentioning insulin signaling [88], post-translational localization of circadian clock components [89] and lipids $[90,91]$ and glycogen metabolism regulation [92]. Moreover, the CFLAR gene encodes the cFLIP protein involved in the inhibition of Fas-mediated apoptosis [93], and the FSTL1 gene plays a role in the immune inflammatory signaling and fibrosis in the liver [94].

Pigs homozygous for the derived $A$-allele of the rs319154814 SNP showed a consistent downregulation of the hepatic mRNA expression of the CFLAR, PPP1CC, $S D C 1, S F 3 A 3$, FSTL1, NAA50 and ELAVL1 genes (Fig. 5), a result that was confirmed by qPCR for PPP1CC mRNA transcripts $(P$-value $=0.027$, Fig. 6a). Although in the qPCR experiment the CFLAR and SF3A3 genes also showed decreased mRNA levels in pigs with homozygous $A A$ genotypes for the rs319154814 SNP, such associations did not reach statistical significance (Fig. 6a). The significant downregulation of at least one top putative mRNA target (i.e., PPP1CC) might suggest that the rs319154814 $(G / A)$ variant could increase the repressive activity of ssc-miR-326. Indeed, when we assessed, with a dedicated qPCR assay, the expression level of ssc-miR-326 transcripts in the liver tissue, we found that ssc-miR-326 $\log _{2}$ relative expression was increased by $\sim 1.9$-fold in pigs homozygous for the $A$-allele, although this result was not statistically significant $(P$-value $=0.178$, Fig. $6 \mathrm{~b})$.

As previously discussed, the $\operatorname{rs} 319154814(G / A)$ polymorphism is located in the apical loop region of sscmiR-326. Although the apical region of a miRNA does not have a function as critical as the seed, polymorphisms located in this specific region can have relevant effects on the structural conformation of the pre-miRNA hairpin. Indeed, Fernandez et al. [19] described a mutation in the apical loop of hsa-miR-30c $(G / A)$ that induces a steric disruption of the pri-miRNA folding structure of the hairpin, hence creating a bulge around the flanking downstream CNNC motif that facilitates the SRSF3 factor accessibility to the RNA sequence [95]. In other words, SNPs in the apical region can modify the efficiency with which the Drosha processing machinery is recruited. The $\mathrm{rs} 319154814(G / A)$ polymorphism detected in our study might have structural consequences similar to those described for the hsa-miR-30c apical loop variant [19].

One clear limitation of our experimental design is that we did not test the existence of miRNA-mRNA interactions and structural miRNA hairpin modifications 
by using functional assays. Such experiments would be necessary to demonstrate our hypothesis that the rs319154814 $(G / A)$ polymorphism in the apical loop of ssc-miR-326 has a role in promoting the processing and maturation efficiency of the hairpin precursor, thus modifying the expression profiles of ssc-miR-326 and its putative mRNA targets.

\section{A polymorphism in the apical loop of microRNA 326 is associated with fatty acid composition traits}

In our study, the only miRNA polymorphism showing significant associations $(q$-value $<0.1)$ with lipid-related phenotypes was the rs319154814 (G/A) SNP in the apical loop of ssc-miR-326. This SNP was associated with myristic fatty acid content in the GM and LD muscles of Duroc pigs, as well as with PUFA/MUFA ratio and gadoleic fatty acid content in LD. To the best of our knowledge, no direct effect of ssc-miR-326 on the metabolism of myristic fatty acid has been described so far, but there are reports suggesting that several of the targets of this miRNA might be involved in carbohydrate and lipid metabolism pathways [96, 97]. For instance, the PPP1CC transcript, one of the predicted targets of miR-326, encodes a subunit of protein phosphatase-1, which activates acetyl-CoA carboxylase $\alpha$ (ACACA) and 6-phosphofructo2-kinase/fructose-2,6-bisphosphatase (PFKFB) enzymes, the main regulators of fatty acid synthesis and glycolysis, respectively [91]. The protein phosphatase- 1 also activates lipogenic transcription factors such as sterol regulatory element-binding protein 1 (SREBF1), carbohydrate-responsive element-binding protein (MLXIPL) and, moreover, it dephosphorylates the DNA-dependent protein kinase encoded by the $P R K D C$ gene, which is another main determinant of hepatic lipogenesis [91]. In summary, a potential effect of the rs319154814 (G/A) SNP on the synthesis or degradation of myristic acid can be envisaged, but such a hypothesis still needs to be confirmed at the functional level. Similarly, Cardoso et al. [98] explored the relationship between miRNA SNPs located in the seed and mature regions of bovine miRNA loci and fatty acid composition traits. By combining lipid-related phenotype data with mRNA and protein abundances in a multi-omics approach, they described three miRNA SNPs that were significantly associated with several unsaturated and polyunsaturated fatty acid traits, as well as with the expression levels of some of their predicted targets [98].

\section{Conclusions}

MicroRNA genes show divergent patterns of variation between Asian and European pigs and wild boars and, in general, they display low levels of polymorphism. As expected, this reduced miRNA variability is particularly accentuated in the seed region, a finding that is likely explained by the strong effects of purifying selection preserving the sequence conservation of this critical site. We detected one SNP in the apical loop of ssc-miR-326 and another one in the precursor region of ssc-miR-23a that are associated with the mRNA expression of several of their putative targets. If confirmed with functional assays, these results would reinforce the need of exploring the role of miRNA variation within and outside the seed in the fine-tuning of mRNA expression in pigs.

\section{Supplementary Information}

The online version contains supplementary material available at https://doi. org/10.1186/s12711-021-00632-3.

Additional file 1: Table S1. List of whole-genome sequences from European domestic pigs ( $E D M, N=40)$, Asian domestic pigs ( $A D M, N=40)$, European wild boars (EWB, $N=20$ ) and Asian wild boars ( $A W B, N=20$ ).

Additional file 2: Table S2. Single nucleotide polymorphisms located in microRNA genes and their frequencies in European (E) and Asian (A) domestic pigs (DM) and wild boars (WB).

Additional file 3: Table S3. Means and standard deviations (SD) of fatness and intramuscular fat content and composition traits recorded in the gluteus medius (GM) and longissimus dorsi (LD) muscles of 345 Duroc pigs.

Additional file 4: Figure S1. Sequence homology between 12 porcine miRNAs harboring SNPs that have been genotyped in the Lipgen population $(\mathrm{N}=345)$ and their corresponding human miRNA orthologous sequences. Figure S2. Number of SNPs present in each of the annotated porcine miRNA loci that display at least one SNP. Figure S3. Venn Diagrams illustrating the sharing of SNPs located at the (a) precursor, (b) mature and (c) seed regions of miRNA loci among Asian domestic pigs (ADM), Asian wild boars (AWB), European domestic pigs (EDM) and European wild boars (EWB). Figure S4. Boxplot distribution depicting whole-genome sequencing statistics for the five Duroc boars that sired the Lipgen population $(\mathrm{N}=345)$.

Additional file 5: Table S4. Set of primers used for the qPCR quantification of three mRNAs putatively targeted by ssc-miR-326.

Additional file 6: Table S5. Distribution of SNPs mapping to specific genomic annotated regions (i.e., protein coding genes, exons, introns, 3'UTR, miRNA, IncRNA, snRNA, snoRNA and pseudogenes) and segregating in the set of whole-genome sequences from 120 Asian and European domestic pigs and wild boars.

Additional file 7: Table S6. List of porcine genes showing 3 or more multi-allelic SNPs mapping to exonic regions in the set of 120 wholegenome sequences from Asian and European domestic pigs and wild boars.

Additional file 8: Table S7. Description of SNPs mapping to porcine mature miRNA loci $(N=409)$ and segregating in the set of 120 wholegenome sequences from Asian and European domestic pigs and wild boars.

Additional file 9: Table S8. Whole-genome sequencing statistics for the five Duroc boars that sired the Lipgen population $(\mathrm{N}=345)$.

Additional file 10: Table S9. List of SNPs mapping to 370 porcine miRNA loci and segregating in the 5 Duroc boars that sired the Lipgen population $(\mathrm{N}=345)$.

Additional file 11: Table S10. Association analyses between SNPs in miRNA genes and the mRNA levels of their predicted targets in the gluteus medius (GM) skeletal muscle and liver tissues of Lipgen pigs. 
Additional file 12: Table S11. Genomic coordinates and sequence of the seed (miRNA) and its predicted 3'UTR binding site (mRNA) for putative miRNA-mRNA pairs showing significant associations after multiple testing correction ( $q$-value $<0.1)$.

Additional file 13: Table S12. Association analyses between miRNA SNPS and the mRNA expression profiles of the whole set of expressed probes/ genes in the porcine gluteus medius (GM) skeletal muscle and liver tissues from Lipgen pigs.

Additional file 14: Table S13. Pathway enrichment analyses of the lists of probes/genes significantly associated at the nominal level $(P$-value $<0.05)$ with miRNA SNP genotypes.

Additional file 15: Table S14. Association analyses between miRNA SNPs and fatness and intramuscular fat content and composition traits recorded in the gluteus medius (GM) and longissimus dorsi (LD) skeletal muscles of 345 Duroc pigs.

\section{Authors' contributions}

MA conceived this study. MA and RQ designed the experimental protocols. RQ coordinated phenotyping recording and contributed to generate microarray expression data. EMS performed the DNA extractions and selected the SNPs to be genotyped. EMS performed all bioinformatic and statistical analyses of the data. MGLS and AC conducted qPCR analyses. DG contributed to population structure analyses. RT performed whole-genome sequencing of the five Duroc boars. EMS and MA drafted the manuscript. All authors read and approved the final manuscript.

\section{Funding}

The research presented in the current publication was funded by Grants AGL2013-48742-C2-1-R and AGL2013-48742-C2-2-R awarded by the Spanish Ministry of Economy and Competitivity. We also acknowledge the support of the Spanish Ministry of Science and Innovation for the Center of Excellence Severo Ochoa 2020-2023 (CEX2019-000902-S) grant awarded to the Centre for Research in Agricultural Genomics (CRAG, Bellaterra, Spain). Emilio Mármol-Sánchez was funded by a FPU Ph.D. grant from the Spanish Ministry of Education (FPU15/01733). María Gracia Luigi-Sierra was funded with a Ph.D. fellowship "Formación de Personal Investigador" (BES-C-2017-0024) awarded by the Spanish Ministry of Economy and Competitivity. Dailu Guan was funded by a Ph.D. fellowship from the Scholarship Council of China (CSC). The authors thank the CERCA Programme of the Generalitat de Catalunya (BarceIona, Spain) for their support and those who provided publicly available data.

\section{Availability of data and materials}

Microarray expression data used in the current study were deposited in the Gene Expression Omnibus (GEO) public repository and are accessible through GEO Series Accession Number GSE115484. Phenotypic and genotypic datasets generated and analyzed during the current study are deposited in the Figshare public repository available at https://figshare.com/projects/SNPs_ miRNA/78690. The whole-genome sequencing dataset from the five Duroc boars is available at the Sequence Read Archive (SRA) database (BioProject: PRJNA626370). Variant Calling Format (VCF) files from the 120 European and Asian pigs belonging to domestic breeds and wild boars, as well as from the five sequenced Duroc boars are available at the following link: https://figshare. com/projects/NCF_PIGs/93140

\section{Declarations}

\section{Ethics approval and consent to participate}

Animal care and management procedures were carried out in accordance with national guidelines for Good Experimental Practices and they were approved by the Ethical Committee of the Institut de Recerca i Tecnologia Agroalimentàries (IRTA).

\section{Consent for publication}

Not applicable.

\section{Competing interests}

The authors declare that they have no competing interests.

\section{Author details}

${ }^{1}$ Centre for Research in Agricultural Genomics (CRAG), CSIC-IRTA-UAB-UB, Universitat Autònoma de Barcelona, 08193 Bellaterra, Spain. ${ }^{2}$ Animal Breeding and Genetics Program, Institute for Research and Technology in Food and Agriculture (IRTA), Torre Marimon, 08140 Caldes de Montbui, Spain. ${ }^{3}$ CNAG-CRG, Centre for Genomic Regulation (CRG), Barcelona Institute of Science and Technology (BIST), Barcelona, Spain. ${ }^{4}$ Departament de Ciència Animal i dels Aliments, Universitat Autònoma de Barcelona, 08193 Bellaterra, Barcelona, Spain.

Received: 16 April 2020 Accepted: 15 April 2021

Published online: 04 May 2021

\section{References}

1. Gebert LFR, MacRae IJ. Regulation of microRNA function in animals. Nat Rev Mol Cell Biol. 2019;20:21-37.

2. Bartel DP. Metazoan microRNAs. Cell. 2018;173:20-51.

3. Chen CYA, Shyu AB. Mechanisms of deadenylation-dependent decay. Wiley Interdiscip Rev RNA. 2011;2:167-83.

4. Eichhorn SW, Guo H, McGeary SE, Rodriguez-Mias RA, Shin C, Baek D, et al. mRNA destabilization is the dominant effect of mammalian microRNAs by the time substantial repression ensues. Mol Cell. 2014;56:104-15.

5. Saunders MA, Liang H, LiWH. Human polymorphism at microRNAs and microRNA target sites. Proc Natl Acad Sci USA. 2007;104:3300-5.

6. Mencía A, Modamio-Høybjør S, Redshaw N, Morín M, Mayo-Merino $F$, Olavarrieta $L$, et al. Mutations in the seed region of human miR-96 are responsible for nonsyndromic progressive hearing loss. Nat Genet. 2009;41:609-13.

7. Auyeung VC, Ulitsky I, McGeary SE, Bartel DP. Beyond secondary structure: primary-sequence determinants license pri-miRNA hairpins for processing. Cell. 2013;152:844-58.

8. Roden C, Gaillard J, Kanoria S, Rennie W, Barish S, Cheng J, et al. Novel determinants of mammalian primary microRNA processing revealed by systematic evaluation of hairpin-containing transcripts and human genetic variation. Genome Res. 2017;27:374-84.

9. Clop A, Marcq F, Takeda H, Pirottin D, Tordoir X, Bibé B, et al. A mutation creating a potential illegitimate microRNA target site in the myostatin gene affects muscularity in sheep. Nat Genet. 2006;38:813-8.

10. Moszyńska A, Gebert M, Collawn JF, Bartoszewski R. SNPs in microRNA target sites and their potential role in human disease. Open Biol. 2017;7:170019.

11. Liang H, Li WH. Lowly expressed human microRNA genes evolve rapidly. Mol Biol Evol. 2009;26:1195-8.

12. Xiong P, Schneider RF, Hulsey CD, Meyer A, Franchini P. Conservation and novelty in the microRNA genomic landscape of hyperdiverse cichlid fishes. Sci Rep. 2019;9:13848.

13. Peterson KJ, Dietrich MR, McPeek MA. MicroRNAs and metazoan macroevolution: insights into canalization, complexity, and the Cambrian explosion. BioEssays. 2009;31:736-47.

14. Lee CT, Risom T, Strauss WM. Evolutionary conservation of microRNA regulatory circuits: an examination of microRNA gene complexity and conserved microRNA-target interactions through metazoan phylogeny. DNA Cell Biol. 2007;26:209-18.

15. Luo J, Wang Y, Yuan J, Zhao Z, Jian LU. MicroRNA duplication accelerates the recruitment of new targets during vertebrate evolution. RNA. 2018;24:787-802.

16. Simkin A, Geissler R, McIntyre ABR, Grimson A. Evolutionary dynamics of microRNA target sites across vertebrate evolution. PLoS Genet. 2020;16:e1008285.

17. Li J, Zhang Z. MiRNA regulatory variation in human evolution. Trends Genet. 2013;29:116-24.

18. Sun G, Yan J, Noltner K, Feng J, Li H, Sarkis DA, et al. SNPs in human miRNA genes affect biogenesis and function. RNA. 2009;15:1640-51.

19. Fernandez N, Cordiner RA, Young RS, Hug N, Macias S, Cáceres JF. Genetic variation and RNA structure regulate microRNA biogenesis. Nat Commun 2017:8:15114. 
20. Jin Y, Lee CGL. Single nucleotide polymorphisms associated with microRNA regulation. Biomolecules. 2013;3:287-302.

21. Larson G, Cucchi T, Dobney K. Genetic aspects of pig domestication. In: Rothschild MF, Ruvinsky A, editors. The genetics of the pig. 2nd ed. Wallingford: CABI; 2011. p. 14-37.

22. Ramos-Onsins SE, Burgos-Paz W, Manunza A, Amills M. Mining the pig genome to investigate the domestication process. Heredity. 2014;113:471-84.

23. Penso-Dolfin L, Moxon S, Haerty W, Di Palma F. The evolutionary dynamics of microRNAs in domestic mammals. Sci Rep. 2018;8:17050.

24. Wei W, Wang G, Cheng Y, Yang R, Song J, Huang S, et al. A miR-511-binding site SNP in the $3^{\prime} U T R$ of $/ G F-1$ gene is associated with proliferation and apoptosis of PK-15 cells. In Vitro Cell Dev Biol Anim. 2019;55:323-30

25. Liu R, Deng D, Liu X, Xiao Y, Huang J, Wang F, et al. A miR-18a bindingsite polymorphism in CDC42 $3^{\prime} U T R$ affects CDC42 mRNA expression in placentas and is associated with litter size in pigs. Mamm Genome. 2019;30:34-41.

26. Ma C, Song H, Yu L, Guan K, Hu P, Li Y, et al. MiR-762 promotes porcine immature Sertoli cell growth via the ring finger protein 4 (RNF4) gene. Sci Rep. 2016;6:32783.

27. Stachowiak M, Szydlowski M, Flisikowski K, Flisikowska T, Bartz M, Schnieke A, et al. Polymorphism in $3^{\prime}$ untranslated region of the pig PPARA gene influences its transcript level and is associated with adipose tissue accumulation. J Anim Sci. 2014;92:2363-71.

28. Bartz M, Szydlowski M, Kociucka B, Salamon S, Jeleń HH, Switonski M. Transcript abundance of the pig stearoyl-COA desaturase gene has no effect on fatty acid composition in muscle and fat tissues, but its polymorphism within the putative microRNA target site is associated with daily body weight gain and feed conversion ratio. J Anim Sci. 2013;91:10-9.

29. Shao GC, Luo LF, Jiang SW, Deng CY, Xiong YZ, Li FE. A C/T mutation in microRNA target sites in BMP5 gene is potentially associated with fatness in pigs. Meat Sci. 2011:87:299-303.

30. Bartz M, Koscianska E, Szczerbal I, Nowacka-Woszuk J, Kociucka B, Salamon S, et al. Polymorphism of the porcine miR-30d is associated with adipose tissue accumulation, its fatty acid profile and the ME1 gene expression. Livest Sci. 2015;182:54-7.

31. Lee JS, Kim JM, Lim KS, Hong JS, Hong KC, Lee YS. Effects of polymorphisms in the porcine microRNA MIR206/MIR133B cluster on muscle fiber and meat quality traits. Anim Genet. 2013:44:101-6.

32. Kim JM, Lim KS, Hong JS, Kang JH, Lee YS, Hong KC. A polymorphism in the porcine miR-208b is associated with microRNA biogenesis and expressions of SOX-6 and MYH7 with effects on muscle fibre characteristics and meat quality. Anim Genet. 2015;46:73-7.

33. Chai J, Chen L, Luo Z, Zhang T, Chen L, Lou P, et al. Spontaneous single nucleotide polymorphism in porcine microRNA-378 seed region leads to functional alteration. Biosci Biotechnol Biochem. 2018;82:1081-9.

34. Choi JW, Chung WH, Lee KT, Cho ES, Lee SW, Choi BH, et al. Wholegenome resequencing analyses of five pig breeds, including Korean wild and native, and three European origin breeds. DNA Res. 2015;22:259-67.

35. Kim H, Song KD, Kim HJ, Park W, Kim J, Lee T, et al. Exploring the genetic signature of body size in Yucatan miniature pig. PLoS One. 2015;10:e0121732.

36. Frantz LAF, Schraiber JG, Madsen O, Megens HJ, Cagan A, Bosse M, et al. Evidence of long-term gene flow and selection during domestication from analyses of Eurasian wild and domestic pig genomes. Nat Genet. 2015:47:1141-8.

37. Bosse M, Megens HJ, Madsen O, Crooijmans RPMA, Ryder OA, Austerlitz $F$, et al. Using genome-wide measures of coancestry to maintain diversity and fitness in endangered and domestic pig populations. Genome Res. 2015;25:970-81.

38. Bolger AM, Lohse M, Usadel B. Trimmomatic: a flexible trimmer for illumina sequence data. Bioinformatics. 2014;30:2114-20.

39. Warr A, Affara N, Aken B, Beiki H, Bickhart DM, Billis K, et al. An improved pig reference genome sequence to enable pig genetics and genomics research. Gigascience. 2020:9:giaa051.

40. Li H. Aligning sequence reads, clone sequences and assembly contigs with BWA-MEM. 2013. http://arxiv.org/abs/1303.3997.

41. McKenna A, Hanna M, Banks E, Sivachenko A, Cibulskis K, Kernytsky A, et al. The genome analysis toolkit: a MapReduce framework for analyzing next-generation DNA sequencing data. Genome Res. 2010;20:1297-303.
42. O'Rawe J, Jiang T, Sun G, Wu Y, Wang W, Hu J, et al. Low concordance of multiple variant-calling pipelines: practical implications for exome and genome sequencing. Genome Med. 2013;5:28.

43. Hasan MS, Wu X, Zhang L. Performance evaluation of indel calling tools using real short-read data. Hum Genomics. 2015;9:20.

44. Backes C, Fehlmann T, Kern F, Kehl T, Lenhof H-P, Meese E, et al. miRCarta: a central repository for collecting miRNA candidates. Nucleic Acids Res. 2018;46:D160-7.

45. Kozomara A, Birgaoanu M, Griffiths-Jones S. miRBase: from microRNA sequences to function. Nucleic Acids Res. 2019;47:D155-62.

46. Shen W, Le S, Li Y, Hu F. SeqKit: a cross-platform and ultrafast toolkit for FASTA/Q file manipulation. PLoS One. 2016;11:e0163962.

47. Grimson A, Farh KKH, Johnston WK, Garrett-Engele P, Lim LP, Bartel DP. MicroRNA targeting specificity in mammals: determinants beyond seed pairing. Mol Cell. 2007;27:91-105.

48. Friedman RC, Farh KKH, Burge CB, Bartel DP. Most mammalian mRNAs are conserved targets of microRNAs. Genome Res. 2009;19:92-105.

49. Price AL, Patterson NJ, Plenge RM, Weinblatt ME, Shadick NA, Reich D. Principal components analysis corrects for stratification in genome-wide association studies. Nat Genet. 2006:38:904-9.

50. Mann HB, Whitney DR. On a test of whether one of two random variables is stochastically larger than the other. Ann Math Stat. 1947;18:50-60.

51. Vidal $\mathrm{O}$, Noguera JL, Amills $M$, Varona L, Gil M, Jiménez N, et al. Identification of carcass and meat quality quantitative trait loci in a Landrace pig population selected for growth and leanness. J Anim Sci. 2005;83:293-300

52. Gallardo D, Pena RN, Amills M, Varona L, Ramírez O, Reixach J, et al. Mapping of quantitative trait loci for cholesterol, LDL, HDL, and triglyceride serum concentrations in pigs. Physiol Genomics. 2008;35:199-209.

53. Gallardo D, Quintanilla R, Varona L, Díaz I, Ramírez O, Pena RN, et al. Polymorphism of the pig acetyl-coenzyme A carboxylase a gene is associated with fatty acid composition in a Duroc commercial line. Anim Genet. 2009;40:410-7.

54. Cánovas A, Quintanilla R, Amills M, Pena RN. Muscle transcriptomic profiles in pigs with divergent phenotypes for fatness traits. BMC Genomics. 2010;11:372.

55. Quintanilla R, Pena RN, Gallardo D, Cánovas A, Ramírez O, Díaz l, et al. Porcine intramuscular fat content and composition are regulated by quantitative trait loci with muscle-specific effects. J Anim Sci. 2011;89:2963-71

56. González-Prendes R, Mármol-Sánchez E, Quintanilla R, Castelló A, Zid A, Ramayo-Caldas Y, et al. About the existence of common determinants of gene expression in the porcine liver and skeletal muscle. BMC Genomics. 2019;20:518.

57. Ramayo-Caldas Y, Mármol-Sánchez E, Ballester M, Sánchez JP, GonzálezPrendes R, Amills $M$, et al. Integrating genome-wide co-association and gene expression to identify putative regulators and predictors of feed efficiency in pigs. Genet Sel Evol. 2019;51:48.

58. Gautier L, Cope L, Bolstad BM, Irizarry RA. affy_analysis of Affymetrix GeneChip data at the probe level. Bioinformatics. 2004;20:307-15.

59. Smedley D, Haider S, Durinck S, Pandini L, Provero P, Allen J, et al. The BioMart community portal: an innovative alternative to large, centralized data repositories. Nucleic Acids Res. 2015;43:W589-98.

60. Cayuela JM, Garrido MD, Bañón SJ, Ros JM. Simultaneous HPLC analysis of a-tocopherol and cholesterol in fresh pig meat. J Agric Food Chem. 2003:51:1120-4.

61. Purcell S, Neale B, Todd-Brown K, Thomas L, Ferreira MAR, Bender D, et al. PLINK: a tool set for whole-genome association and populationbased linkage analyses. Am J Hum Genet. 2007:81:559-75.

62. Zhou X, Stephens M. Genome-wide efficient mixed-model analysis for association studies. Nat Genet. 2012;44:821-4.

63. Karagkouni D, Paraskevopoulou MD, Chatzopoulos S, Vlachos IS, Tastsoglou S, Kanellos I, et al. DIANA-TarBase v8: a decade-long collection of experimentally supported miRNA-gene interactions. Nucleic Acids Res. 2018:46:D239-45.

64. Leclercq M, Diallo AB, Blanchette M. Prediction of human miRNA target genes using computationally reconstructed ancestral mammalian sequences. Nucleic Acids Res. 2017;45:556-66. 
65. Benjamini Y, Hochberg Y. Controlling the false discovery rate: A practical and powerful approach to multiple testing. J R Stat Soc Ser B. 1995;57:289-300

66. Bindea G, Mlecnik B, Hackl H, Charoentong P, Tosolini M, Kirilovsky A, et al. ClueGO: a Cytoscape plug-in to decipher functionally grouped gene ontology and pathway annotation networks. Bioinformatics. 2009;25:1091-3.

67. Shannon P, Markiel A, Ozier O, Baliga NS, Wang JT, Ramage D, et al. Cytoscape: a software environment for integrated models of biomolecular interaction networks. Genome Res. 2003;13:2498-504.

68. Ballester M, Ramayo-Caldas Y, Revilla M, Corominas J, Castelló A, Estellé $J$, et al. Integration of liver gene co-expression networks and eGWAs analyses highlighted candidate regulators implicated in lipid metabolism in pigs. Sci Rep. 2017;7:46539.

69. Rio DC, Ares M, Hannon GJ, Nilsen TW. Purification of RNA by SDS solubilization and phenol extraction. Cold Spring Harb Protoc. 2010;2010:pdb.prot5438.

70. Livak KJ, Schmittgen TD. Analysis of relative gene expression data using real-time quantitative PCR and the $2^{-\triangle \triangle C T}$ method. Methods. 2001;25:402-8

71. Welch BL. The generalizaton of 'student's' problem when several different population varlances are involved. Biometrika. 1947;34:28-35.

72. Timoneda O, Balcells I, Córdoba S, Castelló A, Sánchez A. Determination of reference microRNAs for relative quantification in porcine tissues. PLoS One. 2012;7:e44413.

73. Paudel Y, Madsen O, Megens HJ, Frantz LAF, Bosse M, Crooijmans RPMA, et al. Copy number variation in the speciation of pigs: a possible prominent role for olfactory receptors. BMC Genomics. 2015;16:330.

74. Dueck A, Ziegler C, Eichner A, Berezikov E, Meister G. MicroRNAs associated with the different human Argonaute proteins. Nucleic Acids Res. 2012;40:9850-62

75. Groenen MAM, Archibald AL, Uenishi H, Tuggle CK, Takeuchi Y, Rothschild $M F$, et al. Analyses of pig genomes provide insight into porcine demography and evolution. Nature. 2012;491:393-8.

76. Frantz LAF, Schraiber JG, Madsen O, Megens HJ, Bosse M, Paudel Y, et al. Genome sequencing reveals fine scale diversification and reticulation history during speciation in Sus. Genome Biol. 2013;14:R107.

77. Steri M, Idda ML, Whalen MB, Orrù V. Genetic variants in mRNA untranslated regions. Wiley Interdiscip Rev RNA. 2020;9:e1474.

78. Bachtiar M, Jin Y, Wang J, Tan TW, Chong SS, Ban KHK, et al. Architecture of population-differentiated polymorphisms in the human genome. PLoS One. 2019;14:e0224089.

79. Omariba G, Xu F, Wang M, Li K, Zhou Y, Xiao J. Genome-Wide analysis of microRNA-related single nucleotide polymorphisms (SNPS) in mouse genome. Sci Rep. 2020;10:5789.

80. Gong J, Tong Y, Zhang HM, Wang K, Hu T, Shan G, et al. Genome-wide identification of SNPs in microRNA genes and the SNP effects on microRNA target binding and biogenesis. Hum Mutat. 2012;33:254-63.

81. He S, Ou H, Zhao C, Zhang J. Clustering pattern and functional effect of SNPs in human miRNA seed regions. Int J Genomics. 2018;2018:2456076.

82. Lewis BP, Burge CB, Bartel DP. Conserved seed pairing, often flanked by adenosines, indicates that thousands of human genes are microRNA targets. Cell. 2005;120:15-20.
83. Bartel DP. MicroRNAs: target recognition and regulatory functions. Cell. 2009;136:215-33.

84. Salomon WE, Jolly SM, Moore MJ, Zamore PD, Serebrov V. Single-molecule imaging reveals that Argonaute reshapes the binding properties of its nucleic acid guides. Cell. 2015;162:84-95.

85. Ruda VM, Chandwani R, Sehgal A, Bogorad RL, Akinc A, Charisse K, et al. The roles of individual mammalian Argonautes in RNA interference in vivo. PLoS One. 2014;9:e101749.

86. Huang V, Zheng J, Qi Z, Wang J, Place RF, Yu J, et al. Ago 1 interacts with RNA polymerase II and binds to the promoters of actively transcribed genes in human cancer cells. PLoS Genet. 2013;9:e1003821.

87. Ludwig N, Leidinger P, Becker K, Backes C, Fehlmann T, Pallasch C, et al. Distribution of miRNA expression across human tissues. Nucleic Acids Res. 2016;44:3865-77.

88. Brady MJ, Saltiel AR. The role of protein phosphatase-1 in insulin action. Recent Prog Horm Res. 2001;56:157-73.

89. Schmutz I, Wendt S, Schnell A, Kramer A, Mansuy IM, Albrecht U. Protein phosphatase 1 (PP1) is a post-translational regulator of the mammalian circadian clock. PLoS One. 2011;6:e21325.

90. Liu X, Song B, Li S, Wang N, Yang H. Identification and functional analysis of the risk microRNAs associated with cerebral low-grade glioma prognosis. Mol Med Rep. 2017;16:1173-9.

91. Wang Y, Viscarra J, Kim SJ, Sul HS. Transcriptional regulation of hepatic lipogenesis. Nat Rev Mol Cell Biol. 2015;16:678-89.

92. Toole BJ, Cohen PTW. The skeletal muscle-specific glycogen-targeted protein phosphatase 1 plays a major role in the regulation of glycogen metabolism by adrenaline in vivo. Cell Signal. 2007;19:1044-55.

93. Ram DR, llyukha V, Volkova T, Buzdin A, Tai A, Smirnova I, et al. Balance between short and long isoforms of CFLIP regulates Fas-mediated apoptosis in vivo. Proc Natl Acad Sci USA. 2016;113:1606-11.

94. Mattiotti A, Prakash S, Barnett P, van den Hoff MJB. Follistatin-like 1 in development and human diseases. Cell Mol Life Sci. 2018;75:2339-54.

95. Hargous Y, Hautbergue GM, Tintaru AM, Skrisovska L, Golovanov AP, Stevenin J, et al. Molecular basis of RNA recognition and TAP binding by the SR proteins SRp20 and 9G8. EMBO J. 2006;25:5126-37.

96. Sebastiani G, Grieco FA, Spagnuolo I, Galleri L, Cataldo D, Dotta F. Increased expression of microRNA miR-326 in type 1 diabetic patients with ongoing islet autoimmunity. Diabetes Metab Res Rev. 2011;27:862-6.

97. Kefas B, Comeau L, Erdle N, Montgomery E, Amos S, Purow B. Pyruvate kinase $M 2$ is a target of the tumor-suppressive microRNA-326 and regulates the survival of glioma cells. Neuro Oncol. 2010;12:1102-12.

98. Cardoso TF, Coutinho LL, Bruscadin JJ, da Silva Diniz WJ, Petrini J, Andrade $B G N$, et al. Multi-omics approach reveals mir-SNPs affecting muscle fatty acids profile in Nelore cattle. Genes. 2021;12:67.

\section{Publisher's Note}

Springer Nature remains neutral with regard to jurisdictional claims in published maps and institutional affiliations.

\footnotetext{
Ready to submit your research? Choose BMC and benefit from:

- fast, convenient online submission

- thorough peer review by experienced researchers in your field

- rapid publication on acceptance

- support for research data, including large and complex data types

- gold Open Access which fosters wider collaboration and increased citations

- maximum visibility for your research: over $100 \mathrm{M}$ website views per year
}

At BMC, research is always in progress.

Learn more biomedcentral.com/submissions 